\title{
Learning Automata and the Commons Game
}

\author{
By \\ Petro Verkhogliad
}

\begin{abstract}
A thesis submitted to
the Faculty of Graduate and Postdoctoral Affairs

in partial fulfilment of

the requirements for the degree of

Master of Computer Science

Ottawa-Carleton Institute for Computer Science

School of Computer Science

Carleton University

Ottawa, Ontario
\end{abstract}

June 2011

(C) Copyright

2011, Petro Verkhogliad 


\section{Library and Archives \\ Canada \\ Published Heritage Branch}

395 Wellington Street Ottawa ON K1A ON4 Canada
Bibliotheque et

Archives Canada

Direction du

Patrimoine de l'édition

395 , rue Wellington

Ottawa ON K1A ON4

Canada
Your file Votre référence

ISBN: 978-0-494-83184-7

Our file Notre réfárence

ISBN: 978-0-494-83184-7
NOTICE:

The author has granted a nonexclusive license allowing Library and Archives Canada to reproduce, publish, archive, preserve, conserve, communicate to the public by telecommunication or on the Internet, loan, distribute and sell theses worldwide, for commercial or noncommercial purposes, in microform, paper, electronic and/or any other formats.

The author retains copyright ownership and moral rights in this thesis. Neither the thesis nor substantial extracts from it may be printed or otherwise reproduced without the author's permission.

\section{AVIS:}

L'auteur a accordé une licence non exclusive permettant à la Bibliothèque et Archives Canada de reproduire, publier, archiver, sauvegarder, conserver, transmettre au public par télécommunication ou par l'Internet, prêter, distribuer et vendre des thèses partout dans le monde, à des fins commerciales ou autres, sur support microforme, papier, électronique et/ou autres formats.

L'auteur conserve la propriété du droit d'auteur et des droits moraux qui protège cette thèse. $\mathrm{Ni}$ la thèse ni des extraits substantiels de celle-ci ne doivent être imprimés ou autrement reproduits sans son autorisation.
In compliance with the Canadian Privacy Act some supporting forms may have been removed from this thesis.

While these forms may be included in the document page count, their removal does not represent any loss of content from the thesis.
Conformément à la loi canadienne sur la protection de la vie privée, quelques formulaires secondaires ont été enlevés de cette thèse.

Bien que ces formulaires aient inclus dans la pagination, il n'y aura aucun contenu manquant.

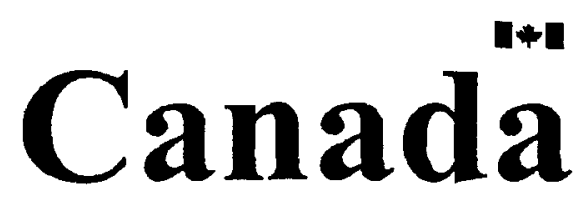




\section{Abstract}

Contention over self-replenishing resources is a common phenomenon which can be simulated via a game of sufficient complexity. The Commons Game provides a framework which allows one to model these types of conflicts.

This work examines the, previously unexplored, subject of application of hillclimbing, particle swarm optimization and a set of learning automata algorithms in order to find the optimal strategies for playing the Commons Game against opponents which use a random action-selection approach.

The obtained results conclusively show that hill-climbing and learning automata are successful at finding optimal strategies for use against random-selection opponents within the confines of the Commons Game. More specifically, we show that learning automata converge to the optimal action in all experimental settings which involve one player using a learning automaton while the remaining players rely on random action-selection. 


\section{Acknowledgments}

I would like to thank Dr. John Oommen, my supervisor, for his inspiration and guidance. His enthusiasm for scientific research is contagious.

I would like to thank my family and friends. This work could not have been completed without their support.

At the same time, I will always be appreciative of all the members, faculty and staff, of the School of Computer Science. 


\section{Contents}

1 Introduction 1

2 Social Dilemmas and the Commons Game 6

2.1 Two-Player Games $\ldots \ldots \ldots \ldots \ldots \ldots \ldots \ldots$

2.2 N-Player Games . . . . . . . . . . . . . . . . . . . . 9

2.3 Commons Game Review . . . . . . . . . . . . . . . . . . . 9

3 Commons Game $\quad 14$

3.1 Commons Game Description . . . . . . . . . . . . . . 14

3.2 Optimal Playing Strategy Against Random Oponents . . . . . . . . . 18

4 Learning Automata $\quad 22$

4.1 A Learning Automaton and its Environment . . . . . . . . . . 23 
4.2 Classification of Learning Automata . . . . . . . . . . . . . . . . 27

4.2.1 Deterministic Learning Automata . . . . . . . . . . . . 27

4.2.2 Stochastic Learning Automata . . . . . . . . . . . . . 28

4.2.2.1 Fixed Structure Learning Automata . . . . . . . 29

4.2.2.2 Variable Structure Learning Automata . . . . . . . 34

4.2.2.3 Descretized Learning Automata . . . . . . . . . 39

$4.2 .3 \quad$ Estimator Algorithms . . . . . . . . . . . . . . . 40

4.2.3.1 Introduction .............. 40

4.2.3.2 Continuous Estimator Algorithms . . . . . . . . . 41

4.2.3.3 Pursuit Algorithm . . . . . . . . . . . 42

4.2.3.4 TSE Algorithm . . . . . . . . . . . . . 43

4.2.3.5 Generalized Pursuit Automaton ........... 44

4.2.4 Discrete Estimator Algorithms . . . . . . . . . . . . . . 45

4.2.4.1 Discretized Pursuit Automaton . . . . . . . . . . 46

4.2.4.2 Discretized TSE Algorithm . . . . . . . . . 47

5 Generic AI Techniques for the Commons Game 50

5.1 Hill Climbing . . . . . . . . . . . . . . . 50 
5.2 Particle Swarm Optimization $\ldots \ldots \ldots \ldots \ldots$

6 Experimental Considerations for AI Solutions $\quad 58$

6.1 Generic AI Methods . . . . . . . . . . . . . . . . . . . 59

6.1 .1 Hill Climbing . . . . . . . . . . . . . . . . . 59

6.1 .2 Particle Swarm Optimization $\ldots \ldots \ldots$. . . . . . 60

6.2 Learning Automata . . . . . . . . . . . . . . . . . . . 60

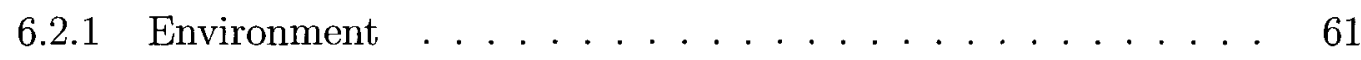

$6.2 .2 \quad$ Fixed Structure Learning Automata . . . . . . . . . . . . 62

6.2.3 Variable Structure Learning Automata . . . . . . . . . . . 64

6.2 .4 Pursuit Automata . . . . . . . . . . . . . 66

6.2 .5 Estimator Automata . . . . . . . . . . . . 66

7 Experimental Results $\quad 67$

7.1 State Range $[8,-8] \ldots \ldots \ldots \ldots \ldots \ldots$

7.2 State Range $[8,8] \ldots \ldots \ldots \ldots \ldots \ldots \ldots$

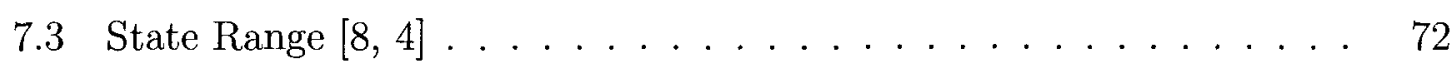

7.4 State Range $[4,0] \ldots \ldots \ldots \ldots \ldots \ldots$ 
7.5 State Range $[0,-4] \ldots \ldots \ldots \ldots \ldots \ldots$

7.6 State Range $[-4,-8] \ldots \ldots \ldots \ldots \ldots \ldots \ldots$

7.7 Results in Games where $N>8 \ldots \ldots \ldots \ldots \ldots$

$\begin{array}{lll}8 & \text { Conclusion } & 77\end{array}$

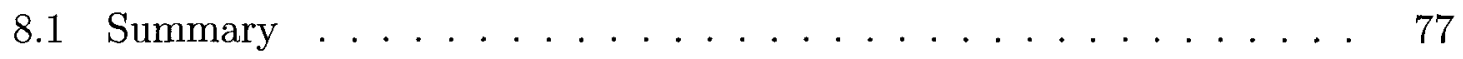

8.2 Future Work . . . . . . . . . . . . . . . . . . . . . 79

$\begin{array}{ll}\text { Bibliography } & 80\end{array}$ 


\section{List of Figures}

4.1 Automaton and Environment interaction. . . . . . . . . . . 25

4.2 The transitions of the Tsetlin $L_{2 N, N}$ automaton on reward (R) and penalty $(\mathrm{P})$ for $N=2 \ldots \ldots \ldots \ldots \ldots$

4.3 The transitions of the Krinsky, $K_{2 N, N}^{1}$, automaton on reward (R) and penalty $(\mathrm{P}) \ldots \ldots \ldots \ldots \ldots \ldots \ldots \ldots \ldots \ldots \ldots \ldots \ldots \ldots \ldots \ldots$

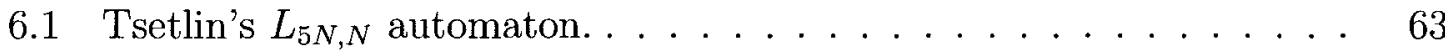

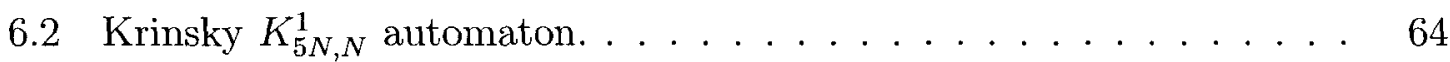




\section{List of Tables}

3.1 Notation used in the description of the Commons Game . . . . . . 15

3.2 Payoff table for an 8 -player group. . . . . . . . . . . . . 18

3.3 Environment replenishment . . . . . . . . . . . . . . 18

3.4 Optimal plays for the Commons Game . . . . . . . . . . . . . . 20

7.1 Results of the various algorithms for the state range $[8,-8] \ldots$. . . 68

7.2 Percentage of time spent in the state range $[8,-8] \ldots \ldots 9$

7.3 Results of the various algorithms for the state range $[8,8] \ldots \ldots 71$

7.4 Results of the various algorithms for the state range $[8,4] \ldots$. . . . 72

7.5 Percentage of time spent in a given state for state range $[8,4] \ldots$. . . 72

7.6 Results of the various algorithms for the state range $[4,0] \ldots 73$

7.7 Percentage of time spent in a given state for the state range $[4,0] \ldots \quad$. 73 
7.8 Results of the various algorithms for the state range $[0,-4] \ldots \ldots 74$

7.9 Percentage of time spent in a given state for the state range $[0,-4]$. . 74

7.10 Results of the various algorithms for the state range $[-4,-8] \ldots$. . . . 75

7.11 Percentage of time spent in a given state for the state range $[-4,-8]$. $\quad 75$

$7.12 L_{R I}$ generated strategies for use in games with 15 players . . . . . . . 76

$7.13 L_{R I}$ generated strategies for use in games with 30 players . . . . . . 76 


\section{Chapter 1}

\section{Introduction}

Since the early days of humanity empires rose by means of easily accessible natural resources. As the population of various nations rose, so did the requirement for the amount of available resources. In fact, most major conflicts in world history can be traced back to one party's desire to acquire a resource from another party. As history progressed, so did the size of the conflicts and their costs. This gave rise to studies on how one should proceed towards ending the conflict in the fastest way possible. One direction taken looked at what can be done when avoiding the conflict is impossible. The other direction, more preventive in its nature, looked at how conflict can be avoided by efficient allocation of available resources. While seemly divergent, these two approaches have very much in common. These similarities have allowed researchers to model both approaches via a game.

While the background for each game may be different, some essential features remain the same. In all games a set of players with different attitudes compete for 
the offered rewards. The goal of every player is to maximize his gain. This gives rise to the following question: With little or no information available about the state of the game, how should one act in order to collect the maximum reward? The desire to answer this question has led to scientists from various fields, such as political science, mathematics, computer science and others, to conduct analyses on different games. In mathematics the study of the optimal behavior and strategy for a game falls into a sub-field known as "game theory". Here, mathematical methods are used to derive and analyze possible ways by which the optimal outcome is attained. From a computer science perspective, analysis of games falls into the sub-field of artificial intelligence (AI). This same sub-field has developed a large number of procedures by which a maximum game reward may be obtained.

Motivated by recent developments surrounding the acquisition of natural resources, such as oil, coal, forest and fish, this work provides a computational exploration of a resource-sharing game known as the Commons Game [56]. All of the essential features, which give rise to dispute, are present in this game. A set of players are given access to a self-replenishing source. Each of them can choose to access it in a manner that is selfish or cooperative. They can also act as a police force or provide additional rewards to other players. Finally, they can completely abstain from resource exploitation. The game framework is structured in a way which makes it difficult to predict the future actions of its participants. Thus, if a computational method were to be applied here it must be one that can cope with the lack of all details about the state of the game.

Some of the earliest AI methods used in gaming were derived from natural human 
behavior. The technique known as Hill-Climbing (HC) [60] takes its name from a process by which a person with limited vision attempts to find the highest nearby peak. It is a simple procedure whereby the individual in question examines the surrounding area and takes a step in the direction deemed to be most promising. The simplicity of this approach is one of the major factors in its popularity. In fact, it is generally the first method used in many analyses where the mathematical foundation has not been well-established or is overly complex. Given that little to no theoretical analysis has previously been done on the Commons Game, hill-climbing is a natural choice for a method by which one can attempt to generate advantageous strategies.

Another popular computational method was created by observing the behavior of flocks of birds and schools of fish. This method, known as Particle Swarm Optimization (PSO) [29], can be perceived as a scheme involving sending out a large number of hill-climbers in search of the same peak. These explorers also have a method by which they can share information about what they have found so far. This makes them more likely to find the target faster and less likely to get trapped by the landscape (as is possible with a single hill-climber). Since PSO is an almost natural extension of hill-climbing it is a good candidate for application to the Commons Game.

While both of the methods presented above have been successful at locating stationary targets, they have been known to generate sub-par results when the target location varies during the course of the search. Developed from a foundation of mathematical psychology, Learning Automata (LA) [38] can be viewed as simple machines designed to work in stochastic environments. They achieve this via communication 
with an oracle/teacher figure. Upon receiving a response from the teacher, the automaton updates its beliefs about the optimal course of action. The automaton then uses its updated data and submits what it believes to be the optimal choice. This process continues until one of the following conditions is met:

1. The automaton learns what the optimal action is.

2. The automaton settles on a sub-optimal action.

3. The automaton runs out of interaction time.

In order to avoid problems associated with items (2) and (3) new learning strategies have been designed. The original finute structure automata [38] gave rise to variable structure automata [38]. In turn, variable structure automata provided the basis for the development of the estimator family of automata [2]. The applicability of this family of algorithms, finite structure, variable structure and estimator automata, to a large variety of problems, has lead us to believe that they would be successful in finding the optimal choice of action in the Commons Game.

The remainder of this work is organized as follows. Chapter 2 gives examples and discusses a selection of 2-player and N-player games, as well as provides a review of recent work done in relation to the Commons Game. Chapter 3 fully describes the Commons Game. This chapter also provides the first contribution of this thesis, which is a computational non-learning approach (Algorithm 3.1) for generating the optimal strategy for use against opponents which employ random action-selection as a means of playing the game. Chapter 4 is a detailed account of the LA used in this work. Chapter 5 provides the description of the modified hill-climbing and particle swam 
optimization algorithms. Chapter 6 describes the method used to generate Commons Game strategies via the algorithms described in Chapters 4 and 5 which can be used against random-selection opponents. Chapter 7 contains the other contribution of this work, the results and discussions of the application of HC, PSO and LA. Finally, Chapter 8 contains the overall conclusions and some directions for future work. 


\section{Chapter 2}

\section{Social Dilemmas and the Commons}

Game

\section{$2.1 \quad$ Two-Player Games}

The Commons Game is an instance of a situation where the many individuallyrational decisions lead to the downfall of the collective. The game was originally developed to model the discussions and actions surrounding the whaling problem. Similar to that, many of the situations and problems facing humanity today can be described and modeled by a social dilemma game.

Most of the work on social dilemma games has been 'focused on the Prisoner's Dilemma $[6,13,23,54,71,72,82]$. The problem is described as follows. Two people are captured after a crime has occurred. The police do not have enough evidence 
to convict either or both of the suspects. Thus, each prisoner is separated from the other and given a choice of testifying against his co-conspirator or staying silent. If one testifies and the other remains silent, the betrayer goes free while the silent individual receives the maximum sentence. If both remain silent, each of them receive the same small sentence. If both of them betray each other, they receive the same medium sentence. The goal is, of course, to spend as little time incarcerated as possible. The question now becomes that of deciding what action each of the prisoners should take.

As an aside, making a purchase from an online retailer or by mail can be thought of as an instance of the Prisoner's Dilemma. The retailer has a choice to send the item being purchased or not. The consumer, on the other hand, has a choice, in case of an Internet purchase to enter the correct billing information, or in the case of the mail/catalogue purchase to send the cheque. If both parties cooperate, the transaction is mutually beneficial. If one party does not follow through, the other one suffers a significant setback. Finally, if both parties defect, both are worse off than if they had followed through.

The best possible outcome for a given player of the Prisoner's Dilemma is to defect while the player remains silent. The next best outcome is mutual cooperation. The next best situation is when both players defect. Finally, the worst situation for a single player occurs when he stays silent and his partner betrays him.

A number of games, used in game theoretic literature, can be derived from the game presented above. The Assurance Game or Stag Hunt [65] is another popular game of similar form. This game describes a conflict between safety and social cooperation. The original description of the game was provided by Jean-Jacques Rousseau, 
who wrote of a situation in which two persons go out on a hunt. Each person can choose to hunt a stag or a hare. Each player must choose an action without knowing the choice of the other. If one player hunts a stag than he will require the help of the second player in order to earn the reward. A single player can hunt a hare on his own. However, the reward for the hare is smaller than that for a stag.

Unlike the Prisonner's Dilemma, the Stag Hunt has two optimums. One occurs when both players cooperate. The other occurs when both players defect.

Another popular game arises if the value of mutual defection is less than the value of mutual cooperation. This situation is known as the game of Chicken [49, 57]. The game can be seen in many Hollywood films. It comprises of two people driving their vehicles towards one another on a collision path. The first person to turn away from the collision path is labeled "chicken". The person who remains on the path is declared the victor. Similar to the Assurance Game, there are two equilibria: complete defection and complete cooperation.

Of the three games presented above, only one has a dominating strategy. In both, the Assurance Game and the Chicken Game, the choice made by the other player is the deciding factor in determining the final outcome. In the Assurance Game both parties want to make the same choice, while in the Chicken Game the opposite choices are wanted. This gives rise to the question whether the same will occur in the Commons Game. In other words, if the majority of players use random selection, does that force the remaining player to utilize the same strategy. 


\section{$2.2 \quad$ N-Player Games}

Although two-player games have enjoyed most of the attention of the research community, a significant amount of work has been done on N-player games $[4,36,37,67,68]$. Generally two groups of N-player games have been recognized based on how the rewards and penalties are allocated. In the first type, referred to as the "social fence" [51], the individual is presented with a situation in which an action has an immediate cost that generates a benefit that is shared by all. Each player has the incentive to avoid the cost, but if cveryone attempts to do so, all are worse off. In the second type, referred to as a "social dilemma" [18, 31], the individual is presented with an opportunity for immediate reward that produces a cost that is shared by all players. In this case, if everyone plays for a reward, the collective result is a large penalty. In real-world situations, the first type of $\mathrm{N}$-player games models the provision of public goods. The second type, and the one that this work focuses on, models the "tragedy of the commons".

\subsection{Commons Game Review}

The earliest mention of the "tragedy of the commons" situation is credited to the Greek philosopher Aristotle (Politics, Book II, Chapter 3). However, the popularization of the dilemma is generally credited to an article published by Hardin in 1968 [24]. In his work, Hardin described the following: a group of cattle herders share access to a piece of grazing land (the commons). The more cattle each herder has on the land, the more reward he receives. However, if there are too many cattle on 
the pasture, it begins to degrade. Thus, the damage to the commons is shared by all participants. Logging, fishing and many other activities that deal with natural resources can all be used as examples of the "tragedy of the commons".

The Commons Game $[55,56]$ represents a situation that is very similar to that described by Hardin $[24,25,26]$. The development of the game was motivated by the conflicting actions and opinions surrounding whaling. At one point, whales were plentiful and the practice of whaling was profitable for all participating nations. Since then, technological improvements have created a more efficient means of finding, killing and processing of whales. Initially, the size of the catches increased. However, after WW II, the whale population and with it the catch size began to decrease. At this stage, the parties involved realized that the rate of exploitation had been too high, and that restrictions had to be put in place. Similar to Hardin's example, it is obviously in the interest of the herders and nations to ensure that the commons remain in a good state. And while it is in everyone's interest to ensure a steady state of the commons, each party involved must trust the other to act responsibly when an agreement on use is put in place. At the time of the creation of the game, Japan and Russia were responsible for $85 \%$ of the world's whale catch [56]. The U.S. and several other nations had declared a moratorium on whaling which left Russia and Japan a larger supply to divide among themselves. Both of these countries believed that if one restricted the catch sizes the other would not and thus continued with high rate of exploitation. This led the authors of the Commons Game to what they believe to be two essential features of the commons: 
- An individual's short-term selfish actions are in conflict with his long-term best interests;

- Each individual's actions affect the others in the commons.

These features are the same as those previously described by Hardin, and serve as the core of the Commons Game. Players have the option to play selfishly or cooperatively. If most of the player-actions are selfish the commons is quickly depleted. Thus, everyone receives less and less of the commons than they would if they had played cooperatively. One the other hand, if the players are cooperative, each of them takes less on every turn. However, with cooperative play all parties can continue using the resource indefinitely.

The differentiating features of the Commons Game as described by Powers [56], from that of Hardin, are three additional actions. The first new action is a withdrawal from the use of the commons. In this case the player earns a constant amount which is significantly less than what he would have received if he had played cooperatively. This action represents the lack of trust on the part of the withdrawing player towards the rest of the community. The second alternative is the ability to punish other players. In this case, the aggressive players suffer significant penalties to their score. Those punishing them also incur a small penalty. This action resembles international sanctions used to prevent the overuse of the resource. The third and final alternative is to reward cooperative players. Thus, players who respect the environment get a small bonus, and those rewarding them incur a small penalty. This could be viewed as a purely altruistic action of giving away some of previously collected resources for the global good. 
Similar to the Prisoner's Dilemma, the Assurance Game and the Chicken Game, significant amount of work has been done on the analysis of the Commons Game. The first results on the use of the game in a classroom setting were reported by Powers in 1983 [55]. The study described in the 1983 paper was directed at finding out how much students learned from repeated-trial social-dilemma games. It is important to note that the first potentially useful strategies are found in this work. The first simulations of the game using computers were performed and documented by Baba et $a l$. in 1984 [7]. While the actual rules of the game were implemented in software, the actual game playing was carried out by students. Baba reported that the students found the use of software both helpful and interesting in helping to understand the commons problem. Continuing from their initial experiments on using computers to help popularize the commons dilemma Baba et al., in their $1999[8,9,10]$ work studied the question of "What would make the Commons Game more exciting to play?". The second paper marks the first attempt to design intelligent players for use in the Commons Game. It is important to note that the fitness of the players was evaluated in the following manner:

1. Players using all available actions (punishment or reward of other players, abstention from the game, cooperative or selfish use) are more desirable than those who only play cooperatively or selfishly.

2. Games where the ranks of the players change frequently are more desirable.

3. Games that spend a large number of turns in the state of environmental depletion are not desirable. 
As can be seen in the above, the players do not necessarily aim to win the game at any cost. The possibility of evolving a mediocre player is quite strong given that the ranks of players should change frequently, and the fact that players are being forced to use the altruistic and punishing actions.

The Commons Game has also been studied from several other directions. More specifically, Kirts [30] used the game to teach students about the frustrations and complexity of managing the commons properly. O'Neill [42] provided the first networkaware implementation of the game in order to study the same question as Kirts. Work by Ohnuma [41] focused on the "free rider" problem within the confines of the Commons Game. At the same time, Faysse [21] provided an analysis of the different variations of commons-types games. Recently, Verkhogliad and Oommen have provided some initial results on strategy discovery in the Commons Game $[79,80]$.

The next chapter describes the details and configuration of the Commons Game. 


\section{Chapter 3}

\section{Commons Game}

\subsection{Commons Game Description}

The Commons Game, designed by Powers et al. [56], was created in an attempt to simulate the workings of a common-pool resource where no single player controls the resource but in which all of the players can use it.

Powers et al. define the game to be playable by groups of 6 to 50 players. Each player, at every turn, has a choice to use the commons selfishly or cooperatively. At the same time, each player has an option to withdraw from the use of the commons, in case the commons are depleted or the player does not trust the others. Another alternative is the punishment action, which penalizes the players who overuse the commons. Finally, each player can reward those participants who use the commons responsibly. 
All of the actions described above are mapped onto five playing cards: green, red, yellow, black and orange. The description of the cards and their respective point allocations $^{1}$ are given below.

\begin{tabular}{|c|l|}
\hline$N_{p}$ & Number of player participating in the game \\
\hline$N_{r}$ & Number of players who played the red card during a given turn \\
\hline$N_{g}$ & Number of players who played the green card during a given turn \\
\hline$N_{y}$ & Number of players who played the yellow card during a given turn \\
\hline$N_{o}$ & Number of players who played the orange card during a given turn \\
\hline$N_{b}$ & Number of players who played the black card during a given turn \\
\hline \hline$t$ & The current turn number \\
\hline$m_{t}$ & Marker position at turn $t$ \\
\hline$S_{t}$ & Commons state at turn $t$ \\
\hline$I\left(S_{t}\right)$ & Replenishment value at state $S_{t}$ \\
\hline
\end{tabular}

Table 3.1: Notation used in the description of the Commons Game

\section{Green card}

The green card symbolizes the maximum utilization of the commons. Players using this card earn the maximum number of points in that environmental state. However, they may receive a score of -20 points if someone plays the black card in the same turn.

\section{Red card}

The red card represents careful or cooperative use of the commons. The players using this card receive a reward worth approximately $40 \%$ of the green card. Red card players also benefit from the orange card plays, receiving 10 extra points for every orange card played in that round. It is important to note

\footnotetext{
${ }^{1}$ Although we consider specific numeric score values as defined in the original manual [56], the principles presented here work even if one changes the values so as to preserve the main properties of the game.
} 
that players playing red cards cannot be penalized by any actions of the other players.

\section{Yellow card}

The yellow card represents abstention from the use of the commons. Each yellow card receives 6 points regardless of the state of the environment or the number of players in the game.

\section{Black card}

The black card can be used to reprimand the players who abuse the environment by playing green. This card is particularly effective when the environment is in a severely degraded state and some player(s) prevents it from replenishing by playing green. The scoring for the black card depends on the number of players in the game. More specifically, it is defined by $-N_{p} / N_{b}$, where $N_{b}$ is the number of black cards played in that round, and $N_{p}$ is the number of participants.

\section{Orange card}

The orange card can be use to encourage the players who respect the environment. As previously mentioned, it gives +10 points to the red card players. However, the orange card player receives a score of $-N_{p} / N_{o}$, where $N_{o}$ is the number of orange cards played during the round, and $N_{p}$ is the number of participants. The reward of the orange card is negative as this action is seen as giving away some of the previously collected materials to another player.

The state of the environment is crucial to determining the reward of the green and red card players. The states range from -8 to +8 . At the start of the game, the 
environment is at state 0 . Table 3.2 shows the scoring table used to calculate the scores for red and green plays for states $+8,+4,0,-4$ and -8 .

The depletion and replenishment of the environment are modeled using a marker, $m$, the value of which ranges from 0 to 180 . At the end of every turn, the marker is updated using Equation (3.1), where $m_{t+1}$ is the marker value in the next turn, $N_{g}$ is the number of green cards played in the current turn, $S_{t}$ is the current state number, $I\left(S_{t}\right)$ is the replenishment value in the given state, and $t$ is the current turn number.

$$
m_{t+1}= \begin{cases}m_{t}-N_{g}+I\left(S_{t}\right) & \text { if } t \bmod 6=0 \\ m_{t}-N_{g} & \text { if } t \bmod 6 \neq 0 .\end{cases}
$$

The value of the marker is used to determine the state of the environment in the next turn as shown in Equation (3.2):

$$
S_{t+1}= \begin{cases}0 & \text { if } 80 \leq m_{t} \leq 100 \\ \frac{m_{t}-90}{10} & \text { if } m_{t}<80 \text { or } m_{t}>100 .\end{cases}
$$

In the interest of clarification, consider the following example of score calculation in which the number of players, $N_{p}$, is 8 , and the state of the environment, $S_{0}$, is 0 . The marker, $m$, has a value of 100 . The current turn, $t$, is 0 . The scoring table is shown in Table 3.2. Five players use the red card $\left(N_{r}=5\right)$, two players use the green 
card $\left(N_{g}=2\right)$, and one player uses the black card $\left(N_{b}=1\right)$. Each of the red card players receive 44 points. The player using the black card receives -8 points. Instead of receiving 106 points, the green card players suffer a penalty of -20 points due to black cards having been played. Since two green cards were played, $m=98$. The game then continues in a similar fashion.

\begin{tabular}{|c|c|c|}
\hline \multicolumn{3}{|c|}{ State +8} \\
\hline$N_{r}$ & $R_{r}$ & $R_{g}$ \\
\hline 0 & - & 200 \\
\hline 1 & 90 & 202 \\
\hline 2 & 90 & 202 \\
\hline 3 & 90 & 202 \\
\hline 4 & 92 & 204 \\
\hline 5 & 94 & 206 \\
\hline 6 & 96 & 208 \\
\hline 7 & 98 & 210 \\
\hline 8 & 100 & - \\
\hline
\end{tabular}

\begin{tabular}{|c|c|c|}
\hline \multicolumn{3}{|c|}{ State +4} \\
\hline$N_{r}$ & $R_{r}$ & $R_{g}$ \\
\hline 0 & - & 186 \\
\hline 1 & 83 & 188 \\
\hline 2 & 83 & 188 \\
\hline 3 & 83 & 188 \\
\hline 4 & 85 & 190 \\
\hline 6 & 87 & 192 \\
\hline 7 & 89 & 194 \\
\hline 8 & 91 & 196 \\
\hline 8 & 93 & - \\
\hline
\end{tabular}

\begin{tabular}{|c|c|c|}
\hline \multicolumn{3}{|c|}{ State 0} \\
\hline$N_{r}$ & $R_{r}$ & $R_{g}$ \\
\hline 0 & - & 100 \\
\hline 1 & 40 & 102 \\
\hline 2 & 40 & 102 \\
\hline 3 & 40 & 102 \\
\hline 4 & 42 & 104 \\
\hline 5 & 44 & 106 \\
\hline 6 & 46 & 108 \\
\hline 7 & 48 & 110 \\
\hline 8 & 50 & - \\
\hline
\end{tabular}

\begin{tabular}{|c|c|c|}
\hline \multicolumn{3}{|c|}{ State -4} \\
\hline$N_{r}$ & $R_{r}$ & $R_{g}$ \\
\hline 0 & - & 14 \\
\hline 1 & -3 & 16 \\
\hline 2 & -3 & 16 \\
\hline 3 & -3 & 16 \\
\hline 4 & -2 & 18 \\
\hline 5 & 1 & 20 \\
\hline 6 & 3 & 22 \\
\hline 7 & 5 & 24 \\
\hline 8 & 7 & - \\
\hline
\end{tabular}

\begin{tabular}{|c|c|c|}
\hline \multicolumn{3}{|c|}{ State -8} \\
\hline$N_{r}$ & $R_{r}$ & $R_{g}$ \\
\hline 0 & - & 0 \\
\hline 1 & -10 & 2 \\
\hline 2 & -10 & 2 \\
\hline 3 & -10 & 2 \\
\hline 4 & -8 & 4 \\
\hline 5 & -6 & 6 \\
\hline 6 & -4 & 8 \\
\hline 7 & -2 & 10 \\
\hline 8 & 0 & - \\
\hline
\end{tabular}

Table 3.2: Payoff table for an 8-player group.

\begin{tabular}{|c|c|c|c|c|c|c|c|c|c|c|c|c|c|c|c|c|c|}
\hline State & 8 & 7 & 6 & 5 & 4 & 3 & 2 & 1 & 0 & -1 & -2 & -3 & -4 & -5 & -6 & -7 & -8 \\
\hline Improvement & 4 & 4 & 5 & 5 & 6 & 6 & 7 & 7 & 8 & 7 & 7 & 6 & 6 & 5 & 5 & 4 & 4 \\
\hline
\end{tabular}

Table 3.3: Environment replenishment

\subsection{Optimal Playing Strategy Against Random Opo-}

\section{nents}

The complexity of the Commons Game does not lend itself to easy mathematical analysis. However, it possible to enumerate all possible plays in a given state and calculate the average value of playing a single card in that state. The result of this procedure is a set of optimal card selections for every state of the game. 
Algorithm 3.1 shows the calculation of the average reward for every card in every play.

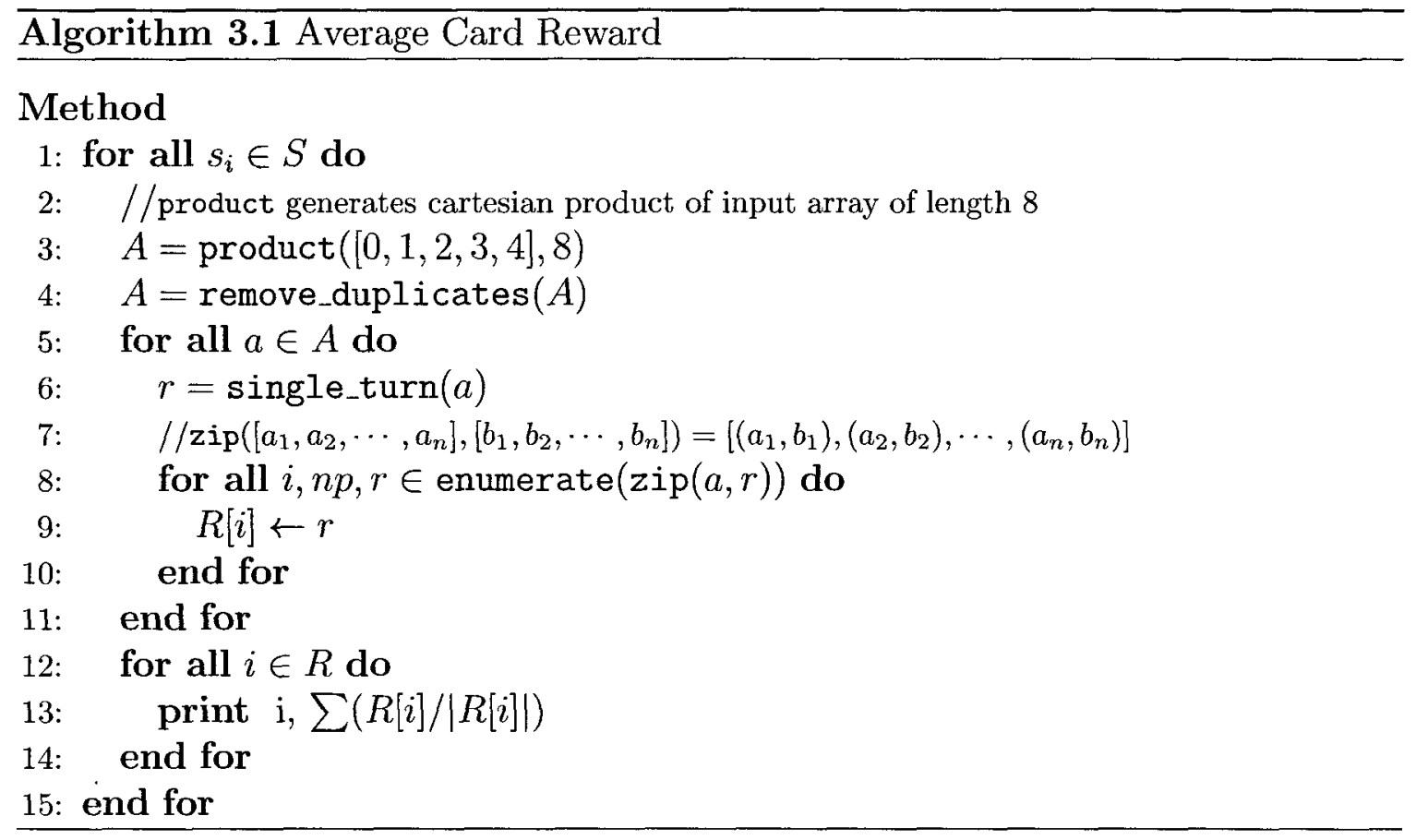

The output of Algorithm 3.1 is shown in Table 3.4. There are several things of note here. First of all, the description of the game shows that the green card returns the highest reward (provided there are no black cards being played) in all states. However, one can see that in some states, the green card has, on average, the worst reward value (states: $-8,-7,-6,-5)$. The game purposely traps any player who continues to overuse the commons without regard for its well-being.

The same theme can be observed by turning one's attention to the rewards for the yellow card. The constant 6 points is the only positive value in states $-8,-7$ and -6 . Thus, the overall theme of forcing players into bettering the environment is observed here as well. Abstention from use by some of the players decreases the number of 


\begin{tabular}{|c|c|c|c|c|c|c|c|c|c|}
\hline Card/State & 8 & 7 & 6 & 5 & 4 & 3 & 2 & 1 & 0 \\
\hline \hline Red & $\mathbf{9 7 . 1 2 7 2}$ & 96.1272 & $\mathbf{9 5 . 1 2 7 2}$ & 94.1272 & 90.1272 & 84.1272 & 74.1272 & 62.1272 & 47.1272 \\
\hline Green & 60.7212 & 59.9939 & 59.2666 & 58.5393 & 55.6303 & 51.2666 & 43.9939 & 35.2666 & 24.3575 \\
\hline Yellow & 6.0 & 6.0 & 6.0 & 6.0 & 6.0 & 6.0 & 6.0 & 6.0 & 6.0 \\
\hline Orange & -3.5596 & -3.5596 & -3.5596 & -3.5596 & -3.5596 & -3.5596 & -3.5596 & -3.5596 & -3.5596 \\
\hline Black & -4.8575 & -4.8575 & -4.8575 & -4.8575 & -4.8575 & -4.8575 & -4.8575 & -4.8575 & -4.8575 \\
\hline
\end{tabular}

\begin{tabular}{|c|c|c|c|c|c|c|c|c|}
\hline Card/State & -8 & -7 & -6 & -5 & -4 & -3 & -2 & -1 \\
\hline \hline Red & -2.8727 & -1.8727 & -0.8727 & 0.1212 & 4.1272 & 10.1272 & 20.1272 & 32.1272 \\
\hline Green & -12.0060 & -11.2787 & -10.5515 & -9.8242 & -6.9151 & -2.5515 & 4.7212 & 13.44 \\
\hline Yellow & 6.0 & 6.0 & 6.0 & 6.0 & 6.0 & 6.0 & 6.0 & 6.0 \\
\hline Orange & -3.5596 & -3.5596 & -3.5596 & -3.5596 & -3.5596 & -3.5596 & -3.5596 & -3.5596 \\
\hline Black & -4.8575 & -4.8575 & -4.8575 & -4.8575 & -4.8575 & -4.8575 & -4.8575 & -4.8575 \\
\hline
\end{tabular}

Table 3.4: Optimal plays for the Commons Game

green cards played, and therefore allows the environment to self-replenish.

Possibly the most interesting result of this experiment is the reward for the red card. Red card plays, on average, return the highest reward in thirteen out of seventeen states. At the same, these rewards are always at least 1.5 times higher than those provided by the green card. The number of points returned by the red card is also interesting. Table 3.2 shows that, for example, in state 8 the minimum red card reward is 90 , while the highest is 100 . Table 3.4 , on the other hand, shows that the average reward is 97.12 . This value is not surprising if one takes into account the orange card. The average reward to the player using the orange card is minimal. However, this action greatly benefits those who choose to use the red card, by increasing their reward.

The observations above allow us to formulate an optimal strategy, shown in Equation (3.3), for use against individuals who employ random action-selection as a means of playing the Commons Game. An astute reader will note that this approach to the game is not valid if there are no random-strategy opponents present. It is easy to see 
that in a game where 7 players use the Equation (3.3) strategy, the last (i.e., the 8th) player, after observing the actions of the other players over the past few turns, can choose the green card for the duration of the game. In this case there is no chance of punishment for the green-card player, while the received rewards will be maximal.

$$
\text { selection }= \begin{cases}\text { red } & \text { if } S_{t} \in\{8, \cdots,-3\} \\ \text { yellow } & \text { if } S_{t} \in\{-3, \cdots,-8\}\end{cases}
$$

This overview of the Commons Game is sufficient for the research done in the subsequent chapters. 


\section{Chapter 4}

\section{Learning Automata}

Learning automata ${ }^{1}(\mathrm{LA})$ are best described by the following quote:

... are adaptive decision making devices suited for operations in unknown environments $\cdots[38]$.

The development of LA theory originated in the field of mathematical psychology, when Bush and Mosteller [14], and Atkinson et. al. [5] described it as a means of modeling observed behavior. In 1961 Tsetlin, a Russian mathematician, studied deterministic LA [76]. Following his initial work, Varshavskii and Vorontsova [78] introduced the families of stochastic variable structure LA. Further significant experimental and theoretical contributions in the field were and are being made by Narenda, Thathachar, Laskshmivarahan, Obaidat, Najim, Baba, Mason, Papadimitriou, and Oommen among others.

\footnotetext{
${ }^{1}$ The discussion in this chapter is based on [46] with the permission of the first author.
} 
Learning automata and the field of learning in stochastic environments has a large variety of applications which include solutions to networking, communications [50], routing [39], network call admission [12], traffic control [77], quality-of-service routing [81], distributed scheduling [66], training hidden Markov models [28], neural network training [69], graph partitioning [47] and classification [11].

In the following sections, we briefly explore the types of LA reported, as well as the different schemes of learning that they employ.

\subsection{A Learning Automaton and its Environment}

The general learning automaton is described by its quintuple which consists of a set of states, a set of outputs/actions, an input, a function that maps the current state and input pair to the next, state and, finally, a function that maps the same current state to the output. More formally, LA is $\langle A, B, Q, F(\cdot, \cdot), G(\cdot)\rangle$ where:

- $A=\left\{\alpha_{1}, \alpha_{2}, \alpha_{3}, \cdots, \alpha_{r}\right\}$ is the set of actions/outputs and $\alpha(t)$ is the action chosen by the automaton at any time instant $t$.

- $B=\left\{\beta_{1}, \beta_{2}, \beta_{3}, \cdots, \beta_{m}\right\}$ is the set of inputs to the automaton. $\beta(t)$ in the input at time instant $t$. In most experiments and theoretical analyses $m=2$ and $B=\{0,1\}$. In this configuration $\beta=0$ represents the automaton being rewarded, while $\beta=1$ represents the automaton being penalized.

- $Q=\left\{q_{1}, q_{2}, q_{3}, \cdots, q_{s}\right\}$ is the set of states where $q(t)$ denotes the state of the automaton at time instant $t$. 
- $F(\cdot, \cdot): Q \times B \rightarrow Q$, the transition function, is a mapping of state and input at time instant $t$ such that $q(t+1)=F(q(t), \beta(t))$ determines the state of the automaton at time instant $t+1$. The transition function can be either stochastic or deterministic.

- $G(\cdot): Q \rightarrow A$ is the output function that determines the output of the automaton at at the same time instant as $\alpha(t)=G(q(t))$. Unlike the transition function, without loss of generality, the output function is always deterministic.

If should be noted that if the sets $A, B$ and $Q$ are finite, the automaton is said to be finite.

As stated before the LA operates within a given environment. The environment for the automaton is described by the triple $\langle A, B, C\rangle$. The definitions of $A, B$ and $C$ are as follows:

- $A=\left\{\alpha_{1}, \alpha_{2}, \alpha_{3}, \cdots, \alpha_{r}\right\}$ is the set of actions. This is the same set as the one that appears in the definition of the automaton.

- $B=\left\{\beta_{1}, \beta_{2}, \beta_{3}, \cdots, \beta_{m}\right\}$ is the set of outputs of the environment. In the following section we consider the case when $m=2$, where $\beta=0$ represents a reward and $\beta=1$ represents a penalty.

- $C=\left\{c_{1}, c_{2}, c_{3}, \cdots, c_{r}\right\}$ is the set of penalty probabilities, where element $c_{i} \in C$ corresponds to an input action $\alpha_{i} \in A$.

The process of learning, the interaction between the automaton and its environment is illustrated in Figure 4.1. The LA choses its action and submits this choice to 


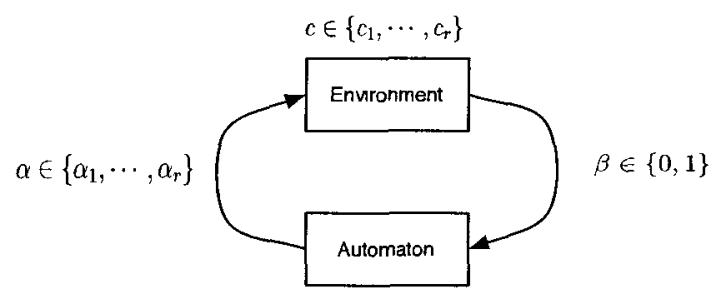

Figure 4.1: Automaton and Environment interaction.

the environment. The environment responds either with a reward or a penalty. The LA's next action depends on the response from the environment. The learning process continues in this fashion until the automaton has submitted a sufficient number of actions to learn the optimal action within the constraints of the current environment. Using the definitions above, the detailed description of this process is is follows. The environment offers the automaton a set of possible actions $\left\{\alpha_{1}, \alpha_{2}, \alpha_{3}, \cdots, \alpha_{r}\right\}$. In turn, the automaton chooses one of the offered actions, $\alpha_{\imath}$, which becomes the next input to the environment. Since the environment is aware of the underling penalty probabilities for all the actions, it replies with either reward $(\beta=0)$ or penalty $(\beta=1)$ depending on the penalty probability $c_{\imath}$. The reward/penalty information guides the LA to select its next action. This process continues until the LA learns the optimal action.

We now provide several definitions which allow us to speak about the "quality" of the automaton. Here $P(t)$ denotes the action probability vector where

$$
P(t)=\left[p_{1}(t), p_{2}(t), \cdots, p_{r}(t)\right]^{T} \text { such that } \sum_{i=1}^{r} p(i)(t)=1 \quad \forall t .
$$


Given an action probability vector, $P(t)$ at time $t$, the average penalty is defined as

$$
\begin{aligned}
& M(t)=E[\beta(t) \mid P(t)]=\operatorname{Pr}[\beta(t)=1 \mid P(t)] \\
& M(t)=\sum_{i=1}^{r} \operatorname{Pr}\left[\beta(t)=1 \mid \alpha(t)=\alpha_{i}\right] \operatorname{Pr}\left[\alpha(t)=\alpha_{i}\right] \\
& M(t)=\sum_{i=1}^{r} c_{i} p_{i}(t) .
\end{aligned}
$$

The average penalty for the pure-chance/random-selection automaton is given by

$$
M_{0}=\frac{1}{r} \sum_{i=1}^{r} c_{i}
$$

As $t \rightarrow \infty$, if the average penalty $M(t)<M_{0}$, at least asymptotically, the automaton is considered to be better than the pure-chance automaton. $E[M(t)]$ is defined as

$$
E[M(t)]=E\{E[\beta(t) \mid P(t)]\}=E[\beta(t)] .
$$

A learning automaton that performs better than the pure-chance automaton is said to be expedient, as shown in Equation (4.7).

$$
\lim _{t \rightarrow \infty} E[M(t)]<M_{0}
$$


An automaton is said to be absolutely expedient if

$$
E[M(t+1) \mid P(t)]<M(t),
$$

meaning that

$$
E[M(t+1)]<E[M(t)] .
$$

Following the above, an automaton is considered optimal if

$$
\lim _{t \rightarrow \infty} E[M(t)]=c_{l} \text { where } c_{l}=\min _{i}\left\{c_{i}\right\} .
$$

Finally, an automaton is considered $\epsilon$-optimal if by setting some parameter defining its structure

$$
\lim _{n \rightarrow \infty} E[M(t)]<c_{l}+\epsilon,
$$

where $\epsilon>0$ is an arbitrarily small constant.

\subsection{Classification of Learning Automata}

\subsubsection{Deterministic Learning Automata}

The automaton is deemed deterministic if both the transition function, $F(\cdot)$, and the output function, $G(\cdot)$ are deterministic. As such, any future state of the automaton can be specified provided the subsequent states and inputs are given. 


\subsubsection{Stochastic Learning Automata}

The automaton is termed stochastic if the transition function $F(\cdot)$ or the output function $G(\cdot)$ are stochastic. In this type of automata, even if the current state and input are known, the subsequent states cannot be fully listed. $F(\cdot)$, in this case, provides the probabilities of reaching different states from the state in question. If $F^{\beta_{1}}, F^{\beta_{2}}, \cdots, F^{\beta_{m}}$ denote the conditional probability matrices $F^{\beta}$ for $(\beta \in B)$ is a $s \times s$ matrix, whose arbitrary element $f_{i j}^{\beta}$ is

$$
f_{i j}^{\beta}=\operatorname{Pr}\left[q(t+1)=q_{j} \mid q(t)=q_{i}, \beta(t)=\beta\right] ; \quad i, j=1,2, \cdots, s .
$$

Each element $f_{i j}^{\beta}$ of the matrix $F^{\beta}$ represents the probability of the automaton moving from state $q_{i}$ to the state $q_{j}$ upon receiving an input $\beta$ from the environment. $F^{\beta}$ is a Markov matrix and therefore

$$
\sum_{j=1}^{s} f_{i j}^{\beta}=1, \text { where } \beta \in B ; i=1,2, \cdots s .
$$

Similarly if $G(\cdot)$ is stochastic then

$$
g_{i j}^{\beta}=\operatorname{Pr}\left[\alpha(t)=\alpha_{j} \mid q(t)=q_{\imath}\right], i \in\{1, \cdots, s\} \text { and } j \in\{1, \cdots, r\}
$$

where $g_{i j}$ represents the elements of the conditional probability matrix of dimension $s \times r . g_{i j}$ denotes the probability that the automaton will choose action $\alpha_{j}$ when in state $q_{i}$. Similar to the above we have:

$$
\sum_{j=1}^{r} g_{i j}=1, \text { for each row } i=1,2, \cdots, s .
$$




\subsubsection{Fixed Structure Learning Automata}

If the conditional probabilities $f_{i j}^{\beta}$ and $g_{i j}$ do not vary with each time step $t$ and the input sequence, the automaton is described as Fixed Structure Stochastic Automaton (FSSA). The most popular examples of this type of automata are due to Tsetlin [76], Krylov [33] and Krinsky [32]. All of the automata listed above are $\epsilon$-optimal2 ${ }^{2}$.

Tsetlin proposed several different LA designs. His first design, termed $L_{2,2}$ has two states $\left\{\phi_{1}, \phi_{2}\right\}$, and two inputs $\left\{\alpha_{1}, \alpha_{2}\right\}$. The automaton accepts inputs from a set $\{1,0\}$ and switches upon encountering an input of 1 (corresponding to a penalty), and remains in the same state on the input of 0 (corresponding to a reward). The transition matrices for this automaton are as follows:

$$
F(0)=\left[\begin{array}{ll}
1 & 0 \\
0 & 1
\end{array}\right] \quad F(1)=\left[\begin{array}{ll}
0 & 1 \\
1 & 0
\end{array}\right]
$$

The environment is described by a set of penalty probabilities $\left\{c_{1}, c_{2}\right\}$ where $c_{i}$ is the probability of getting a response $\beta=1$ from the environment given the input $\alpha_{i}$.

The pure chance automaton operating in the same environment would select the actions with equal probability. Thus,

$$
M_{0}=\frac{1}{2}\left(c_{1}+c_{2}\right)
$$

${ }^{2}$ The Tsetlin automaton is $\epsilon$-optimal only if $\min \left\{c_{1}, c_{2}, \cdots, c_{r}\right\}=c_{l} \leq \frac{1}{2}$. 
Further analysis shows that

$$
\lim _{n \rightarrow \infty} M(n)=\frac{2 c_{1} c_{2}}{c_{1}+c_{2}}=M\left(L_{2,2}\right)
$$

If $c_{1} \neq c_{2}$ then

$$
\frac{2 c_{1} c_{2}}{c_{1}+c_{2}}<\frac{1}{2}\left(c_{1}+c_{2}\right)
$$

implying that the $L_{2,2}$ automaton is expedient but not $\epsilon$-optimal.

Following the $L_{2,2}$ automaton, Tsetlin suggested a modification that, provided certain constraints on the environment, can achieve optimality. Named $L_{2 N, 2}$ by Tsetlin, this automaton has $2 N$ states and 2 actions and attempts to incorporate the past behavior of the system in its decision rule for choosing the sequence of actions. While the $L_{2,2}$ automaton switches from one action to the other on encountering a failure, $L_{2 N, 2}$ keeps an account of the number of successes and failures received for each action. When the number of failures is greater than the number of successes the automaton switches from one action to the other.

More specifically $L_{2 N, 2}$ has $2 N$ states, $\left\{\phi_{1}, \phi_{2}, \cdots, \phi_{2 N}\right\}$ and two actions $\left\{\alpha_{1}\right.$ and $\left.\alpha_{2}\right\}$. The states $\left\{\phi_{1}, \cdots, \phi_{N}\right\}$ correspond to action $\alpha_{1}$ while states $\left\{\phi_{N+1}, \cdots, \phi_{2 N}\right\}$ correspond to action $\alpha_{2}$. Therefore,

$$
G\left[\phi_{\imath}\right]=\alpha_{1} \quad i=1,2, \cdots, N
$$

and

$$
G\left[\phi_{l}\right]=\alpha_{2} \quad i=N+1, \cdots, 2 N
$$


If the automaton is in a state $\phi_{i}(1 \leq i \leq N)$, it proceeds with the action $\alpha_{1}$. If the result of this action is negative the state changes as shown below.

$$
\phi_{i} \rightarrow \phi_{i+1} \text { and } \phi_{N} \rightarrow \phi_{2 N}
$$

If the response is positive then the state transitions are as follows:

$$
\phi_{i} \rightarrow \phi_{i-1} \text { and } \phi_{1} \rightarrow \phi_{1} \text {. }
$$

On the other hand, the state transitions of the automaton in states $\phi_{i}$ when $N+1 \leq i \leq 2 N$ and action $\alpha_{2}$ is performed, are as follows for a negative response:

$$
\phi_{i} \rightarrow \phi_{i+1} \text { and } \phi_{2 N} \rightarrow \phi_{N}
$$

Shown below are the transitions in case $\alpha_{2}$ results in a positive response.

$$
\phi_{i} \rightarrow \phi_{i-1} \text { and } \phi_{N+1} \rightarrow \phi_{N+1} .
$$

The above transition definitions are also shown in Figure 4.2. Finally, if $\min _{i}\left\{c_{i}\right\} \leq \frac{1}{2}$, the expected penalty for the $L_{2 N, 2}$ automaton is

$$
\lim _{N \rightarrow \infty} M\left(L_{2 N, 2}\right)=\min \left(c_{1}, c_{2}\right)
$$

This shows that the $L_{2 N, 2}$ automaton with a sufficiently large memory capacity $N$ (for each action), performs almost exclusively the action with the smallest penalty probability. As stated above, this automaton is $\epsilon$-optimal is $\min (c 1, c 2) \leq \frac{1}{2}$. 


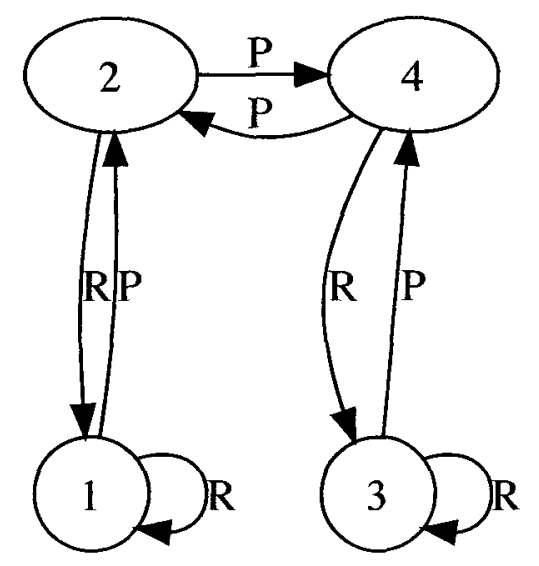

Figure 4.2: The transitions of the Tsetlin $L_{2 N, N}$ automaton on reward (R) and penalty (P) for $N=2$

The second $\epsilon$-optimal FSSA was presented by Krinsky [32]. The transition graph for this automaton behaves exactly like the $L_{2 N, 2}$ automaton when the response of the environment is negative. However, if the response is positive, the LA passes from any state $\phi_{i}(i=1,2, \cdots, N)$ to the state $\phi_{1}$ and from any state $\phi_{i}(i=N+1, N+2, \cdots, 2 N)$ to the state $\phi_{N+1}$.

The expected penalty can be shown to be:

$$
M\left(K_{1}\right)=\frac{c_{1} c_{2}^{N}+c_{2} c_{1}^{N}}{c_{1}^{N}+c_{2}^{N}}=\frac{\frac{1}{c_{1}^{N-1}}+\frac{1}{c_{2}^{N-1}}}{\frac{1}{c_{1}^{N}}+\frac{1}{c_{2}^{N}}} .
$$

As the memory depth $N$ tends to infinity, one can see that

$$
\lim _{N \rightarrow \infty} M\left(K_{1}\right)=\min \left(c_{1}, c_{2}\right) .
$$




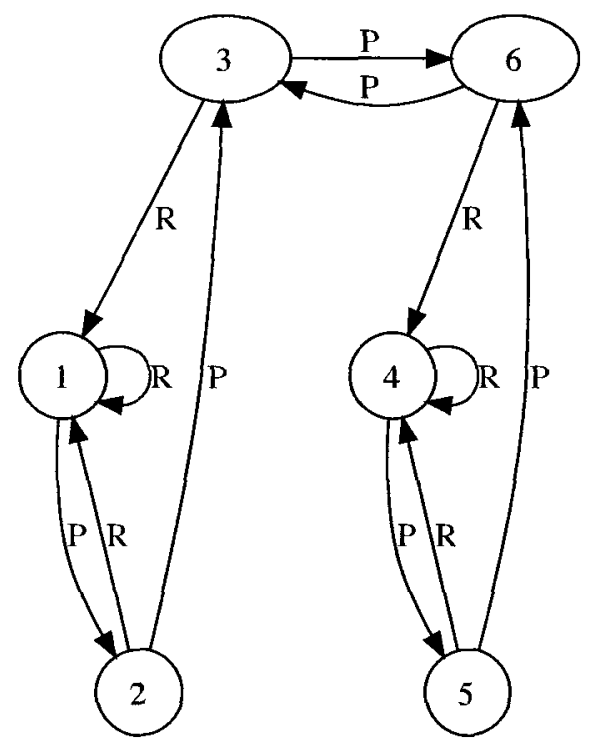

Figure 4.3: The transitions of the Krinsky, $K_{2 N, N}^{1}$, automaton on reward (R) and penalty $(\mathrm{P})$.

Hence, the Krinsky automaton is $\epsilon$-optimal for all stationary random environments.

Another Soviet mathematician, Krylov [33], developed an improvement to the Krinsky automaton. For the most part Krylov's automaton is similar to that of the Krinsky and Tsetlin. The state transitions are identical to the $L_{2 N, 2}$ automaton if the response from the environment is positive. In the case when the response is negative, the LA in a state $\phi_{i}(i \neq 1, N, N+1,2 N)$ passes to a state $\phi_{i+1}$ with probability $\frac{1}{2}$ and to a state $\phi_{i-1}$ with a probability $\frac{1}{2}$.

When $i=1$ or $N+1, \phi_{i}$ stays in the same state with probability $\frac{1}{2}$ and moves to $\phi_{i+1}$ with the same probability. When $i=N$, the LA in $\phi_{N}$ moves to $\phi_{N-1}$ and $\phi_{2 N}$, each with probability $\frac{1}{2}$ and similarly, when $i=2 N$, the LA in $\phi_{2 N}$ moves to $\phi_{2 N-1}$ 
and $\phi_{N}$, each with probability $\frac{1}{2}$. The matrix $F(1)=f_{i j}^{1}$ is defined by

$$
\begin{array}{rlrl}
f_{i, i-1}^{1} & =f_{i, i+1}^{1}=\frac{1}{2} ; & i \neq 1, N, N+1 \text { and } 2 N \\
f_{11}^{1}=f_{12}^{1}=\frac{1}{2} ; & f_{N, N-1}^{1}=f_{N, 2 N}^{1}=\frac{1}{2} \\
f_{N+1, N+1}^{1}=f_{N+1, N+2}^{1}=\frac{1}{2} ; & f_{2 N, 2 N-1}^{1}=f_{2 N, N}^{1}=\frac{1}{2}
\end{array}
$$

Given that $\lambda_{\alpha}=\frac{c_{\alpha}}{1+d_{\alpha}}(\alpha=1,2)$ the expected penalty for the Krylov automaton is:

$$
M\left(K_{2 N, N}^{2}\right)=\frac{\frac{1}{\lambda_{1}^{N-1}} \frac{\lambda_{1}^{N}-1}{\lambda_{1}-1}+\frac{1}{\lambda_{2}^{N-1}} \frac{\lambda_{2}^{N}-1}{\lambda_{2}-1}}{\frac{1}{c_{1}} \frac{1}{\lambda_{1}^{N-1}} \frac{\lambda_{1}^{N-1}}{\lambda_{1}-1}+\frac{1}{c_{2}} \frac{1}{\lambda_{2}^{N-1}} \frac{\lambda_{2}^{N-1}}{\lambda_{2}-1}}
$$

This gives

$$
\lim _{N \rightarrow \infty} M\left(K_{2 N, N}^{2}\right)=\min \left(c_{1}, c_{2}\right)
$$

In this case the stochastic updating scheme makes the automaton $\epsilon$-optimal in all stationary random environments.

\subsubsection{Variable Structure Learning Automata}

Variable Structure Stochastic Automata (VSSA) are those in which the state transition probabilities are not fixed. In this case, the state transition are updated every time instant using a predefined updating scheme. The transition probability $f_{i j}^{\beta}$ and 
the output function $g_{i j}^{\beta}$ vary with time. The first of the VSSA where introduced by Varshavskii and Vorontsova [78]. At any time instant the action of the VSSA is chosen based on the action probability distribution vector, which is afterwards updated based on the response from the random environment.

A VSSA is defined by a quintuple $\langle Q, A, B, G, T\rangle$ where

- $Q$ represents the states of the automaton.

- $A$ is the set of actions.

- $B$ is the set of possible responses from the environment.

- $G$ is the output function.

- $T$ is the action probability updating scheme,

$$
T:[0,1]^{r} \times A \times B \rightarrow[0,1]^{r},
$$

such that

$$
P(t+1)=T[P(t), \alpha(t), \beta(t)]
$$

where $P(t)$ is the action probability vector.

In general, VSSA can be analyzed using a discrete-time Markov process. However, if the probability updating scheme $T$ is time-invariant, $\{P(t)\}_{t \geq 0}$ is a discretehomogeneous Markov process, where the probability vector at the current time instant is $P(t)$. Therefore, each updating scheme, $T$, identifies a different type of learning algorithm as shown below: 
- Absorbing algorithms are those where the updating scheme contains absorbing states.

- Nonabsorbing algorithms are those where the Markov process does not contain absorbing states.

- Linear algorithms are those where $P(t+1)$ is a linear function of $P(t)$.

- Nonlinear algorithms are those where $P(t+1)$ is a non-linear function of $P(t)$.

In a VSSA, if a selected action $\alpha_{i}$ is rewarded, the probability of the current action is increased. At the same time, the probabilities of the other actions are decreased. If the current action, $\alpha_{i}$ is penalized, then its probability is typically decreased. Subsequently, the probabilities of other actions could be increased. These procedures lead to a set of learning schemes for VSSA:

- Reward-Penalty (RP): The probabilities are updated without regard for the outcome.

- Reward-Inaction (RI): The probabilities are updated if the automaton was rewarded. No updates are performed if the automaton was penalized.

- Inaction-Penalty (IP): The probabilities are updated if the automaton was penalized. No updates are performed if the automaton was rewarded.

The general updating scheme for a VSSA with $r$ actions is shown below in a 
stationary environment with $\beta=\{0,1\}$. The selected action is $\alpha(t)=\alpha_{i}$.

$$
\begin{aligned}
& \text { for } \beta(t)=0, \forall j \neq i, p_{j}(t+1)=p_{j}(t)-g_{j}[P(t)] \\
& \text { for } \beta(t)=1, \forall j \neq i, p_{j}(t+1)=p_{j}(t)-h_{j}[P(t)] .
\end{aligned}
$$

Given that $P(t)$ is a probability vector, $\sum_{j=1}^{r} p_{j}(t)=1$, when $\beta(t)=0$

$$
p_{i}(t+1)=p_{i}(t)+\sum_{j \neq 1, j \neq i}^{r} g_{j}[P(t)]
$$

and when $\beta(t)=1$,

$$
p_{i}(t+1)=p_{i}(t)-\sum_{j \neq 1, j \neq i}^{r} h_{j}[P(t)]
$$

The functions $h_{j}$ and $g_{j}$ are nonnegative and continuous in [0,1], and obey:

$$
\begin{aligned}
& \forall i=1,2, \cdots, r, \forall P \in(0,1)^{R}, \\
& 0<g_{j}(P)<p_{j}, \text { and } \\
& 0<\sum_{j=1, j \neq i}^{r}\left[p_{j}+h_{j}(P)\right]<1 .
\end{aligned}
$$

The following four learning schemes for continuous linear VSSA have been extensively studied:

- Linear reward-inaction scheme $\left(L_{R I}\right)$ 
- Linear inaction-penalty scheme $\left(L_{I P}\right)$

- Symmetric linear reward-penalty scheme $\left(L_{R P}\right)$

- Linear reward- $\epsilon$-penalty scheme $\left(L_{R-\epsilon-P}\right)$.

For a two action learning automaton, let

$$
g_{i}[P(t)]=a p_{j}(t) \text { and } h_{j}[P(t)]=b\left(1-p_{j}(t)\right) .
$$

In Equation (4.38), the reward and penalty parameters, $a$ and $b$ obey $0<a<1$, $a \leq b<1$.

The $L_{R I}$ scheme was first introduced by Norman [40], and then studied by Shapiro [64]. As previously stated, the overall design of this scheme dictates that the action probabilities are updated only when the automaton receives a positive response from the environment.

The probability updating equations for this scheme are shown below:

$$
\begin{aligned}
& p_{1}(t+1)=p_{1}(t)+\alpha\left[1-p_{1}(t)\right], \text { if } \alpha(t)=\alpha_{1}, \text { and } \beta(t)=0, \\
& p_{1}(t+1)=(1-\alpha) p_{1}(t), \text { if } \alpha(t)=\alpha_{2}, \text { and } \beta(t)=0, \\
& p_{1}(t+1)=p_{t}(t), \text { if } \alpha(t)=\alpha_{t} \text { or } \alpha_{2}, \text { and } \beta(t)=1
\end{aligned}
$$

As can be seen from the above updating equations, the $L_{R I}$ scheme has two absorbing states. Thus, the convergence of this scheme is dependent on the initial 
probabilities. For this reason the $L_{R I}$ scheme is not suitable for non-stationary environments. However, for stationary environments this learning scheme is absolutely expedient and $\epsilon$-optimal.

The $L_{I P}$ and $L_{R P}$ schemes are very similar to the $L_{R I}$ scheme. The complete analysis is found in [38].

\subsubsection{Descretized Learning Automata}

Discretized Learning Automata (DLA) can be thought of as a mixture of FSSA and VSSA. This set of algorithms was originally introduced by Thathachar and Oommen [73] as they studied ways to increase the speed convergence of FSSA and VSSA. The properties of DLA, and the updating schemes for action probabilities were further described by Oommen [43, 44].

Discretization refers to restricting the probability of choosing the actions to a small set of values in the $[0,1]$ interval. Therefore, the updating of the actions is performed in steps. DLA possess finite sets just as FSSA do. However, due to the fact that they deal with random vectors, DLA behave similar to VSSA. There are two types of DLA:

- Linear - the action probabilities are evenly spaced over the closed [0,1] interval.

- Nonlinear - the probability values are unevenly spaced over the $[0,1]$ interval. 
Discretization provides two distinct advantages over the previously described algorithms. The first of these is the overall increase in the speed of convergence. Originally, it was assumed that the random number generator could generate real values with arbitrary precision. In the case of DLA, if the action probability is close to unity, the probability of choosing that action increases to unity directly, instead of asymptotically [73]. The second advantage is that continuous VSSA can theoretically take on any value in the $[0,1]$ interval, while all implementations of random number generators are pseudo-random. Thus, the set of possible random values is not truly infinite in $[0,1]$, but finite.

\subsubsection{Estimator Algorithms}

\subsubsection{Introduction}

Development of new LA algorithms is driven by the desire to increase their convergence speed. It is with this goal that the discretized algorithms were developed. Thathachar and Sastry $[63,75]$ eventually developed another set of algorithms, the so-called estimator LA, which have a faster rate of convergence than that of any other family. Similar to the rest of LA designs, this family also maintains and updates an action-probability vector. Unlike previous designs, this set of algorithms also keeps a running estimate of the rewards for each of the available actions. Subsequently, the reward-estimate vector is used in the action-probability updating equations. Typically, the reward-estimate vector is denoted by $\hat{D}(t)=\left[\hat{d}_{1}(t), \cdots, \hat{d}_{r}(t)\right]^{T}$, and the state vector is denoted by $Q(t)=<P(t), \hat{D}(t)>$. 
Estimator algorithms initially pre-process the environment by sampling the response of the environment to each of the actions. This process establishes the initial reward-estimate vector. The use of the reward-estimate vector increases the confidence of the learning automaton in selecting the best action. Non-estimator VSSA schemes update the action-probability vector directly given the response from the environment, increasing the probability of one action while decreasing others depending on the type of scheme used. Estimators, however, perform updates using both the reward-estimate vector and the current response from the environment. Thus, the environment has direct and indirect influence on the action-probability vector. This may lead to increases in probability to non-rewarded actions.

While they are more complicated, estimator algorithms have been shown to be an order of magnitude faster than non-estimators. Furthermore, Lanctot and Oommen [48] have developed discretized estimators, which have a superior speed of convergence in comparison to their non-discretized counterparts.

\subsubsection{Continuous Estimator Algorithms}

Continuous estimator algorithms were originally introduced by Thathachar and Sastry $[63,75]$. Here the probability of choosing an action can be any real number in the interval $[0,1]$. 


\subsubsection{Pursuit Algorithm}

The class of pursuit algorithms is so named since they are designed to pursue the action which the automaton considers optimal at any given time. The first of these algorithms, $C P_{R P}$, developed by Thathachar and Sastry [75], pursues the best action by changing the probability of the current optimal action. The perceived best action is the one that receives an increase, proportional to $1-p_{m}(t)$, in probability. At the same time, the sub-optimal actions are penalized by having their probabilities reduced.

The general operation of this algorithm is as follows. At first, an action $\alpha(t)$ is chosen based on the probability distribution $P(t)$. Regardless of whether the response was a reward or a penalty, the algorithm increases the probability of the action with highest reward estimate, while reducing the probabilities of the other actions. Afterwards, the running estimates of the reward probabilities are updated. The estimate vector $\hat{D}(t)$ is computed using the following formula

$$
\hat{d}_{i}(t)=\frac{W_{i}(t)}{Z_{i}(t)}, \forall i=1,2, \cdots, r,
$$

where $W_{i}(t)$ is the number of times the action $\alpha_{i}$ has been rewarded, and $Z_{i}(t)$ is the number of times $\alpha_{i}$ has been chosen. The formal pursuit algorithm is shown in Algorithm 4.1.

The $C P_{R P}$ algorithm is quite similar to the previously described $L_{R P}$ algorithm. Both update the action-probability vector depending on the response from the environment. However, the key difference between the two algorithms is the following: 
$L_{R P}$ directly updates the action-probability vector, and moves it in the direction of the most recently rewarded action. $C P_{R P}$, on the other hand, moves the probability vector in the direction of the action with the highest reward estimate. Thathachar and Sastry have provided $\epsilon$-optimality proofs. They also have, experimentally, shown that pursuit algorithms are significantly faster and more accurate than the non-pursuit counterparts.

The reward-inaction version of this algorithm is similar in design. It is described in depth in [2].

\subsubsection{TSE Algorithm}

The TSE algorithm was designed by Thathachar and Sastry [74]. Similar to other estimator algorithms, TSE maintains a running reward-estimate vector $\hat{D}(t)$ which is used to update the action-probability vector. If an action $\alpha_{i}(t)$ is rewarded, the components of the action-probability vector with values higher than $\hat{d}_{i}(t)$ are increased. At the same time, the components with values lower than $\hat{d}_{i}(t)$ are decreased. This is implemented via an indicator function, $S_{i j}(t)$. The value of $S_{i j}(t)$ is 1 if $\hat{d}_{i}(t)>\hat{d}_{j}(t)$ and 0 if $\hat{d}_{i}(t) \leq \hat{d}_{j}(t)$. The convergence proofs for this algorithm can be found in [74]. Given the description, we observe that the value of $P(t+1)$ does not directly depend on the response from the environment. Instead, the response from the environment changes the values of the reward estimate vector $\hat{D}(t)$ which, in turn, is used in conjunction with $S_{i j}(t)$. If the $i^{\text {th }}$ action is rewarded, the probability values of the action with reward estimates higher than that of the currently-selected action are updated 
using:

$$
p_{j}(t+1)=p_{j}(t)-\lambda\left\{f\left[\hat{d}_{i}(t)-\hat{d}_{j}(t)\right] \frac{p_{i}(t)-p_{j}(t) p_{i}(t)}{r-1}\right\}
$$

when $\hat{d}_{i}(t)<\hat{d}_{j}(t)$. For the actions with reward estimates smaller than the currently selected action estimate, the updates are performed using

$$
p_{j}(t+1)=p_{j}(t)-\lambda f\left[\hat{d}_{i}(t)-\hat{d}_{j}(t)\right] p_{j}(t)
$$

The full algorithm is shown in Algorithm 4.2.

In their work, Thathachar and Sastry have proven that the TSE algorithm is $\epsilon$ optimal. At the same time, they have shown, experimentally, that the TSE converges several orders of magnitude faster than the $L_{R I}$ algorithm.

\subsubsection{Generalized Pursuit Automaton}

In [2] Agache and Oommen described a generalized version of pursuit automaton $\left(C P_{R P}\right)$. Unlike its predecessor, this algorithm pursues all actions that that possess a reward estimate higher than that of the selected action. It has been, experimentally, shown that this algorithm converges faster than all previous algorithms. The updating scheme for is given by 


$$
\begin{aligned}
& p_{j}(t+1)=(1-\lambda) p_{j}(t)+\frac{\lambda}{K(t)}, \text { if } \hat{d}_{j}(t)>\hat{d}_{i}(t)(t), j \neq i, \\
& p_{j}(t+1)=(1-\lambda) p_{j}(t), \text { if } \hat{d}_{j}(t) \leq \hat{d}_{i}(t), j \neq i \\
& p_{i}\left(t_{1}\right)=1-\sum_{j \neq i} p_{j}(t+1),
\end{aligned}
$$

where $K(t)$ denotes the number of actions that have estimates greater than estimate of the reward of the action currently chosen. For the full description of the algorithm the reader is invited to consult [3].

\subsubsection{Discrete Estimator Algorithms}

Previously it was stated that the discretized versions of the continuous variable structure learning automata have a significantly faster convergence rate. The same can be said about continuous and discretized estimator LA: Lanctot and Oommen [34] have developed a set of discretized estimator algorithms (DEA). Here the components of the action probability vectors are allowed take take on a finite set of discrete values in the interval $[0,1]$, which is divided into a number of sub-intervals proportional to the resolution parameter, $N$. As before, a reward-estimate vector is maintained to keep an estimate of the reward probability of each action.

The same authors have shown that in order for a DEA to be $\epsilon$-optimal, it must have two properties: 
- Moderation property: All DEAs where the magnitude by which an action probability value can decrease is bounded by $\frac{1}{r N}$.

- Monotone property: A DEA is said to possess the monotone property if $\exists$ an integer $N$ such that, for all the resolution parameters $N>N_{0}, p_{m}(t) \rightarrow 1$ with probability 1 as $t \rightarrow \infty$, where $p_{m}(t)$ is the component of largest magnitude in $P(t), m$ is an index and $t_{0}$ is a time instant $t_{0}<\infty$, such that $\hat{d}_{m}(t)>$ $\hat{d}_{j}(t), \forall j$ with $j \neq m$ and $\forall t$ such that $t \geq t_{0}$, where $\hat{d}_{m}(t)$ is the maximal component of $\hat{D}(t)$.

\subsubsection{Discretized Pursuit Automaton}

The discrete pursuit algorithm (DPA) is not vastly different from its continuous counterpart. As in the $C P_{R P}$ algorithm, the DPA algorithm operators in three steps. If $\delta=\frac{1}{r N}$ (where $N$ is the resolution, and $r$ is the number of actions) denotes the smallest step size, the integral multiples of $\delta$ denotes the step sizes in which the action probabilities are updated. Similar to the continuous reward-inaction algorithm, when the chosen action $\alpha(t)=\alpha_{\imath}$ is penalized the action probabilities remain unchanged. If the chosen action $\alpha(t)=\alpha_{\imath}$ is rewarded, and the algorithm has not converged, all actions except the one with the highest reward estimate have their probabilities decreased by integral multiples of $\delta$.

In their work, Lanctot and Oommen [45] have also shown that the DPA algorithm has both of the properties, monotonicity and moderation, required for $\epsilon$-optimality. They have also experimentally shown that the DPA algorithm is at least $60 \%$ faster than the $C P_{R P}$ algorithm. 


\subsubsection{Discretized TSE Algorithm}

As with the DPA, Lanctot and Oommen [34] have also discretized the continuous TSE algorithm, thus creating the discretized TSE algorithm (DTSE). They proved that the DTSE possesses the monotonicity and the moderation properties. Therefore this algorithm is $\epsilon$-optimal.

The discretization of the algorithm introduced two new parameters to the algorithm:

- $\delta=\frac{1}{r N \theta}$, where $\mathrm{N}$ is the resolution parameter.

- $\theta$, an integer which represents the largest amount by which any of the action probabilities can change in a single iteration.

The full description of the DTSE algorithm is found in [34]. 


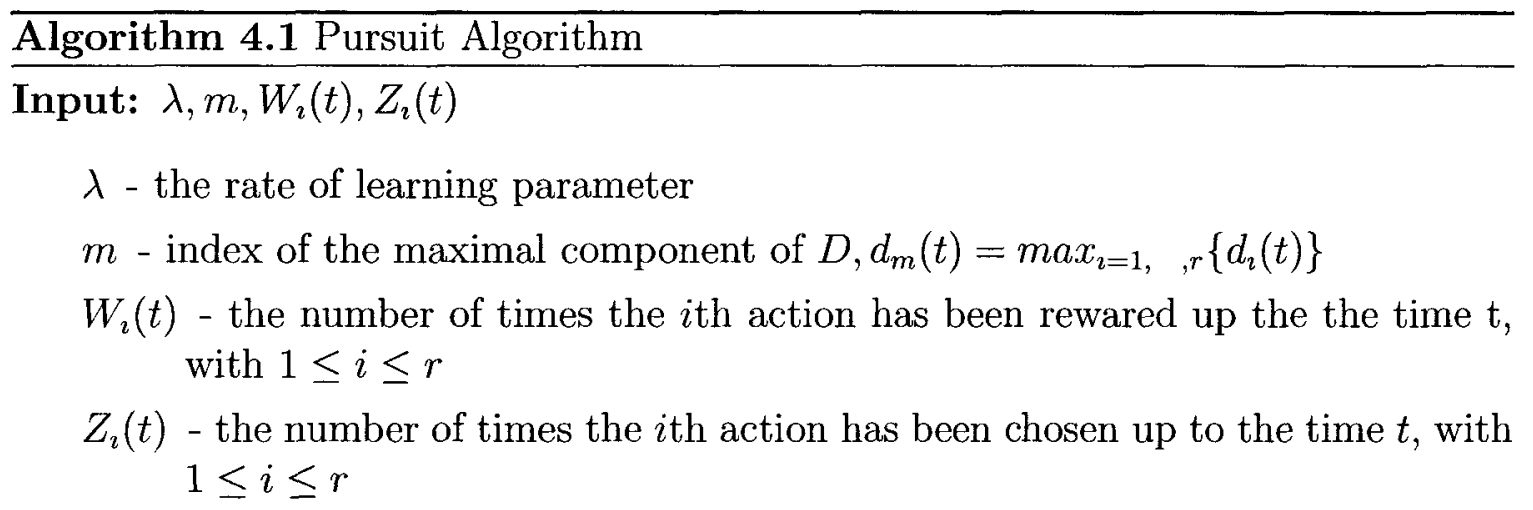

\section{Method}

Initialize: $p_{t}(t), D$

$p_{t}=1 / r$, for $1 \leq i \leq r$

Initialize $D$ by picking each action a small number of times

\section{Repeat}

1: At time $t$ pick $\alpha(t)$ according to probability distribution $P(t)$. Let $\alpha(t)=\alpha(t)$.

2: if is_optrmal_action $\left(\alpha_{m}\right)$ then

3: $\quad p_{m}(t+1)=p_{m}(t)+\lambda\left(1-p_{m}(t)\right)$

4: $\quad p_{\jmath}(t+1)=p_{\jmath}(t)-\lambda p_{\jmath}(t)$

5: end if

6: $W_{\imath}(t+1)=W_{\imath}(t)+(1-\beta(t))$

7: $Z_{\imath}(t+1)=Z_{\imath}(t)+1$

8: $\hat{d}_{\imath}(t+1)=\frac{W_{\imath}(t+1)}{Z_{\imath}(t+1)}$

9: for all $j$ such that $j \neq i$ do

10: $\quad W_{\jmath}(t+1)=W_{\jmath}(t)$

11: $\quad Z_{\jmath}(t+1)=Z_{\jmath}(t)$

12: $\quad d_{\jmath}(t+1)=d_{\jmath}(t)$

13: end for

End Repeat 


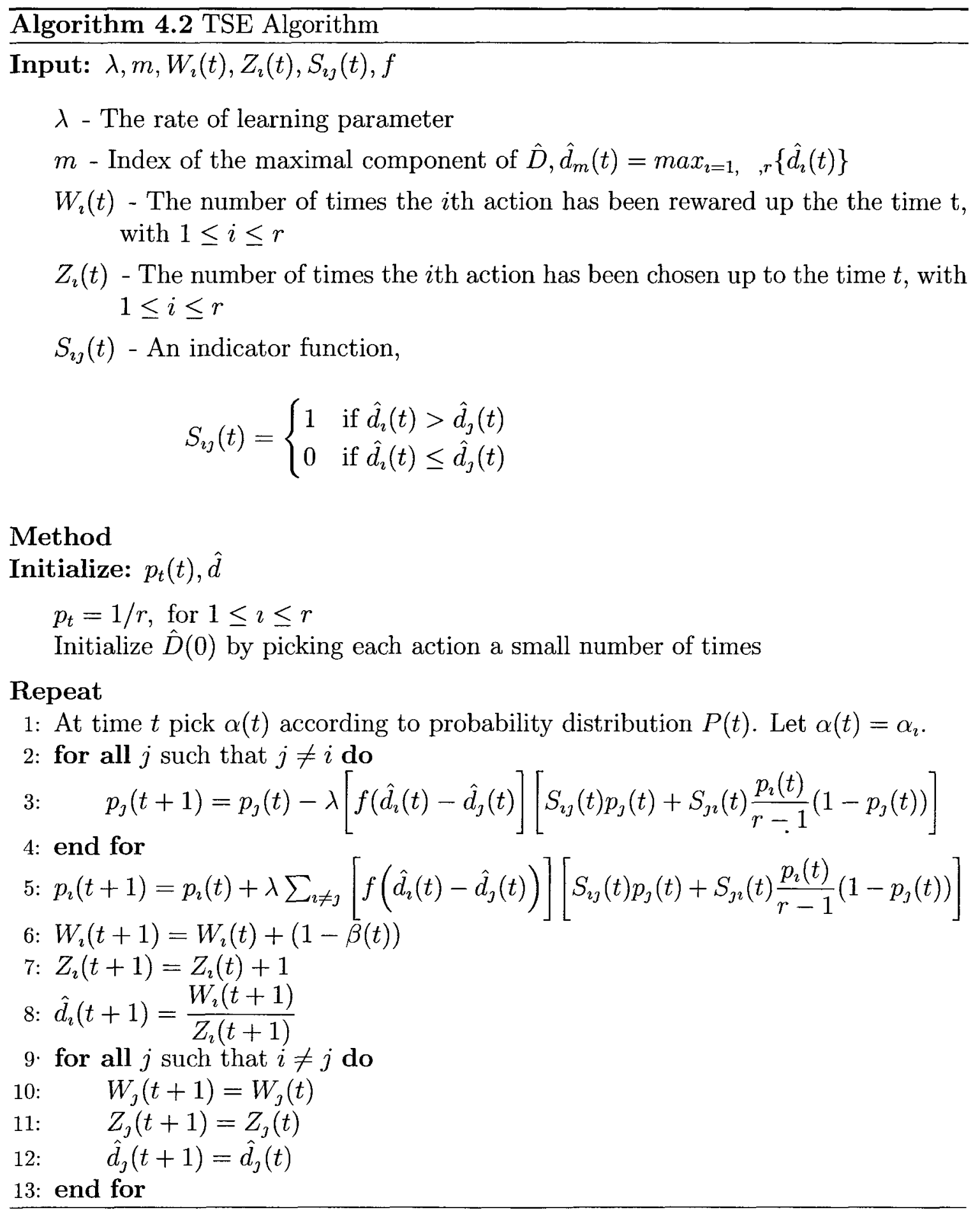




\section{Chapter 5}

\section{Generic AI Techniques for the Commons Game}

As previously stated, there has been little prior work done with regards to the application of computational/AI techniques within the confines of the Commons Game $[79,80]$. Consequently, the choice the investigation techniques, hill climbing and particle swarm optimization, used in this work was inspired by previous work in applications of AI to gaming problems.

\section{$5.1 \quad$ Hill Climbing}

Hill climbing $(\mathrm{HC})$ is one of the earliest and most well-known general purpose optimization techniques. The first application of HC in games dates back to 1959, when it was used to play checkers. [61, 62]. 
In general, $\mathrm{HC}$ starts with either a man-made or a random solution, and attempts to find the optimum by sampling the area around it, and choosing the "best" value as determined by a heuristic function ${ }^{1}$.

There are several different types of hill climbing schemes reported in the literature [60]. The earliest variation is known as "simple hill climbing". This method selects the first candidate solution that is better than the current one, while, as previously mentioned the goodness of a given solution is defined by the heuristic function.

Steepest ascent/descent hill climbing improves upon the initial description by iterating over all the possible candidate solutions, and selecting that one with the highest or lowest heuristic value.

Stochastic hill climbing is yet another variation on the basic algorithm. Here, a solution is selected at random from a set candidate solutions. For a solution to appear in the selection set its heuristic value must be above a pre-set threshold.

Due to the complexity of the Commons Game, we have opted to use another variation of the basic $\mathrm{HC}$ algorithm, shown in Algorithm 5.1. This variation is in a small respect, inspired by learning approaches in reinforcement learning [70], LA [38] and genetic algorithms [22]. The closest neighbour of our algorithn is RMHC proposed by Forrest and Mitchell [22]. This HC algorithm also differs from LA approaches in several ways. First of all, at every time step LA randomly select and use the action based on the action-probability vector. Secondly, as previously discussed, only estimator automata employ an initial testing phase (i.e., to get some some initial

\footnotetext{
${ }^{1}$ Heuristic value of a candidate solution is the value returned by the heuristic function when supplied with a candidate solution.
} 


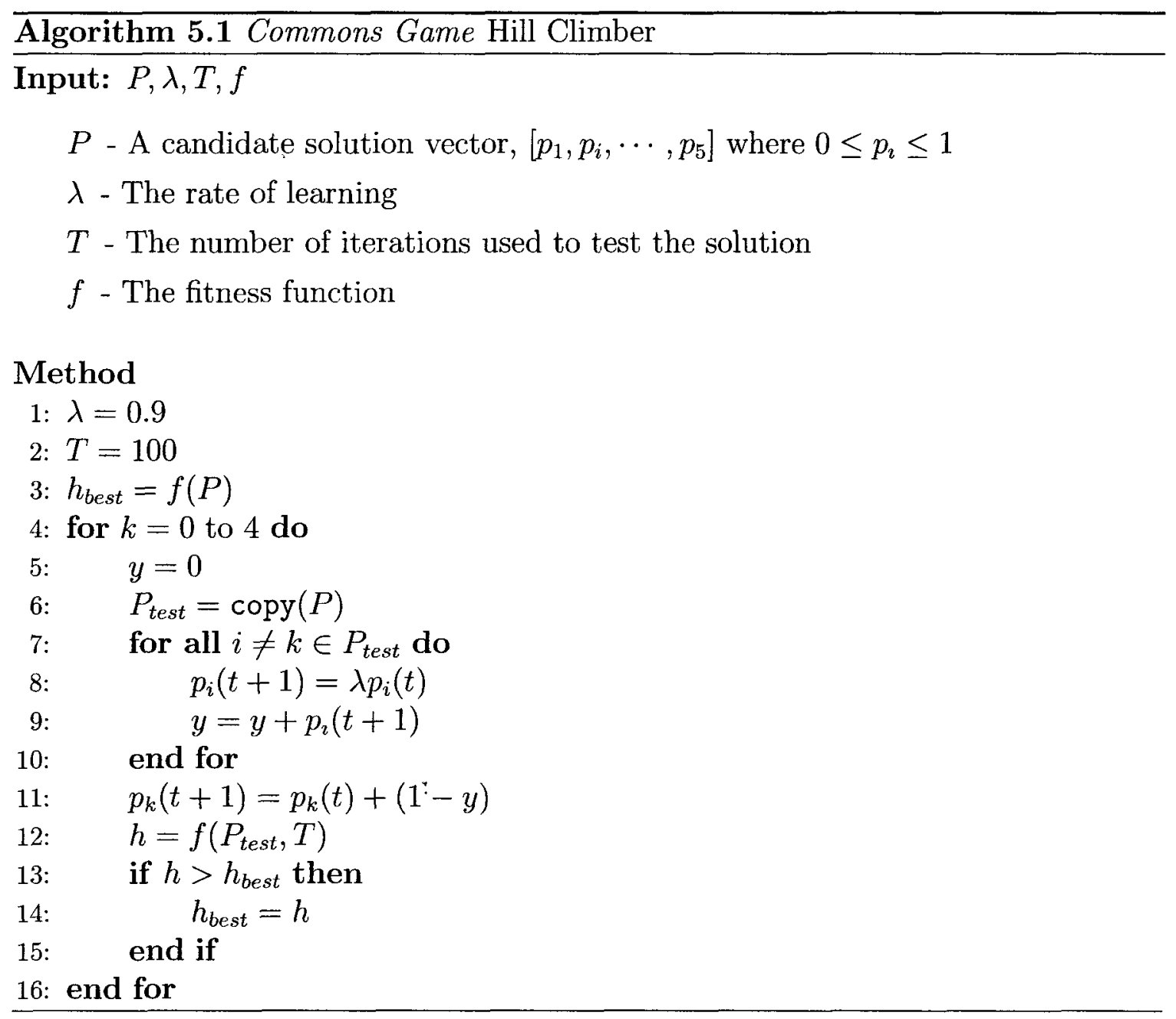

estimates). The $\mathrm{HC}$ algorithm used here does not select the action which currently has the highest probability of reward. Rather, by generating candidate solutions it attempts to find an action with the highest future reward.

At the start of the game the $\mathrm{HC}$ is initialized and added to a game with $N-1$ other players. At each turn, the $\mathrm{HC}$ player updates its probability vector, $P$, in an attempt to increase its overall reward. Thus, at every turn, five candidate vectors are created using the current solution as a template. In each of the vectors the probability of the 
target card, $p_{i}$, is increased by $1-\lambda$ as shown in Equation (5.2), and the probabilities of the other cards are decreased by $\frac{1-\lambda}{4}$ as shown in Equation (5.1). In these equations, $p_{i}(t)$ is the current probability of selecting a card $i, p_{i}(t+1)$ is the probability value that will be used in the next turn, and, $i, j \in\{$ green, red, yellow, orange, black $\}$ such that $i \neq j$.

$$
\begin{aligned}
& p_{i}(t+1)=\lambda p_{i}(t) \\
& p_{j}(t+1)=p_{j}(t)+\left(1-\sum_{i \in P} p_{i}(t+1)\right)
\end{aligned}
$$

Each of the five vectors are then tested by playing $T$ turns of the game. The vector which returns the highest fitness value (i.e., the sum of scores over the $T$ turns) is selected as the current best solution. This process continues for the remainder of the game.

The strategies discovered by this HC scheme are discussed in Chapter 7 .

\subsection{Particle Swarm Optimization}

Particle Swarm Optimization (PSO), is a global optimization, population-based evolutionary algorithm, that has been used in a large variety of applications, in a multitude of fields. This diversity has been best described by Poli [52] in 2008. In his work, Poli separated PSO applications into 26 different categories, such as communication networks, classification, optimization, control, economics, graphics, metallurgy, scheduling and etc. PSO has also been applied to gaming problems $[1,17,35]$. 
One of the earliest attempts to apply an algorithm that eventually became the modern PSO algorithm was in rendering computer animation that resembles natural phenomena. For this task Reeves [58] created a particle system that used simple individuals to resemble a fuzzy object. The system relied upon a series of moving points which were initialized to random positions. During the initialization step each particle was assigned an initial velocity vector. Over the course the simulation, the velocity vectors were adjusted by either predefined or random factors.

The next major development in this area was taken by Reynolds [59], who used Reeves' particle system as the base for his flocking algorithm. Reynolds added particle orientation and inter-object communication. This development removed the requirement for establishing individual direction vectors. However, this raised the question of conflict resolution between single particles. Reynolds resolved the conflict issue deterministically using prioritized ordering.

The basic version of PSO that is most-commonly used is the one developed by Kennedy and Eberhart [29] when they worked on extending Reynolds' model to reflect social behavior. They replaced the so-called "roost" goal, developed by Heppner and Grenander [27], and devised an alternative flocking algorithm with for the task of searching for food.

These developments eventually led to the publication of the canonical PSO algorithm in which a set of $n$-dimensional particles, traveling through the solution space, track their own best solution, velocity, and the global best solution.

Similar to HC, each particle uses a set of updating equations to direct itself and the rest of the swarm toward the optimum. The updating equation for a particle's 
position, $\overrightarrow{x_{i}}$, is shown in Equation (5.3), where $\overrightarrow{v_{i}}$ is the particle's velocity. The updating equation for the latter, is shown in Equation (5.4), where $g$ and $\hat{g}$ are, respectively, the particle's and the swarm's "best" solutions.

$$
\begin{aligned}
& \overrightarrow{x_{i}}(t+1)=\overrightarrow{x_{i}}(t)+\overrightarrow{v_{i}}(t) . \\
& v_{i j}(t+1)=\omega v_{i j}(t)+c_{1} r_{1}\left[g_{i j}(t)-x_{i j}(t)\right]+c_{2} r_{2}\left[\hat{g}_{i j}(t)-x_{i j}(t)\right] .
\end{aligned}
$$

In the above equations, $x_{i j}$ and $v_{i j}$ are the $j^{t h}$ components of $\overrightarrow{x_{i}}(t)$ and $\overrightarrow{v_{i}}(t)$ respectively. Further, $c_{1}$ and $c_{2}$ are weighting coeffients for the local and global best. $\omega$ is the inertia weight. Finally, the acceleration coefficients $r_{1}$ and $r_{2} \in U(0,1)^{n}$. It has been shown that under the following conditions:

$$
\omega>\frac{1}{2}\left(c_{1}+c_{2}\right)-1 ; \text { and } 0<\omega<1,
$$

convergence to a stable equilibrium point is guaranteed. The full, standard PSO algorithm is presented in Algorithm 5.2.

The structure of the particle neighbourhood used in our work is commonly referred to as gbest. This configuration was intentionally selected as this is the first time it has been used in relation to the Commons Game. Futhermore, it has been shown by Carlisle [15] that gbest PSO with 30 particles and with no constraints on the maximum velocity and position can perform very well. At the same time, other PSO variants, noted by Kennedy and Eberhart [19], Engelbrecht [20], Clerc [16] and Poli et al. [53] or more complex approaches for neighbourhood configuration are ideal candidates for future work. 
Each particle in the swarm is initialized with a random position vector which represents a game strategy vector. The PSO player is then added to a game with $N-1$ other players which employ a random action-selection strategy (as before $N=8$ ). At every game turn, the fitness of every particle is evaluated by using it's position to play $T$ turns of a training instance of the game. The fitness value is the sum of the rewards received over the $T$ turns. Similar to the HC approach, the fitness function used does not incorporate any information about the game, except for player's score over the $T$ turns. If the new fitness value is higher than any previously seen value, the new strategy becomes the particle's "best" solution. The individual "best" solutions are then compared to determine the global "best" solution. This continues for the rest of the game. 


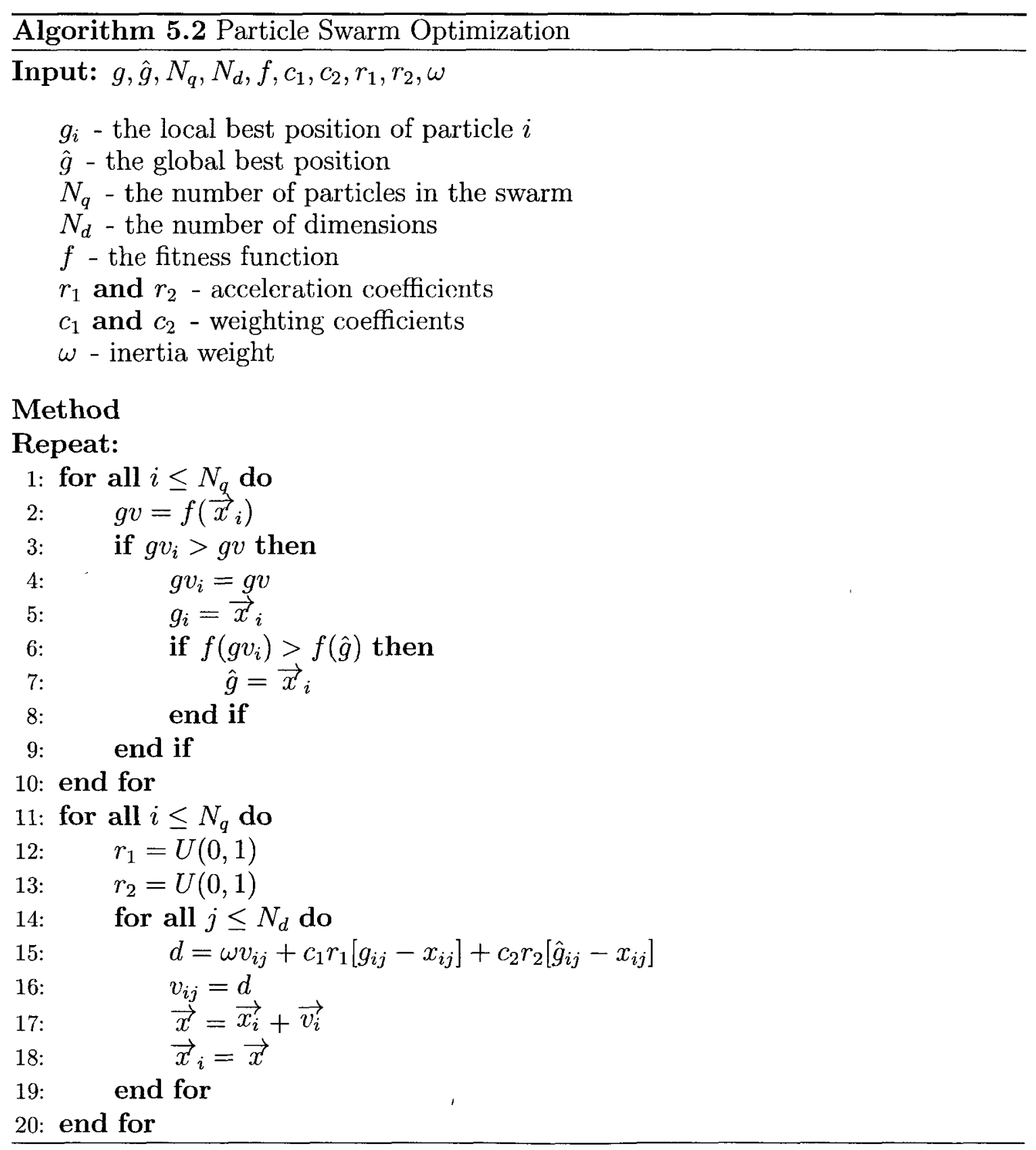




\section{Chapter 6}

\section{Experimental Considerations for}

\section{AI Solutions}

In the previous chapters we presented a general description of two AI methods, $\mathrm{HC}$ and PSO, and of the family of LA. This chapter describes the application details of the above set of algorithms in the confines of the Commons Game.

Although each of the algorithms has different parameters, the same experimental procedure was used to generate the results discussed in Chapter 7. Each algorithm was used in 100 trials of the Commons Game and in 6 different state configurations of the game. Each game involves 1,000 turns, implying that each algorithm was required to select an action, one of the cards, 1,000 times in a single game. 


\subsection{Generic AI Methods}

\subsubsection{Hill Climbing}

As stated earlier, the hill climbing algorithm used in this work is a variation of previously well-established methods. Thus, several parameters had to be initialized prior to $\mathrm{HC}$ participating in an instance of the game.

The first of these is the learning rate parameter, $\lambda$, which controls the amount by which the probabilities of the solution vector, $P$, are updated. The value was arrived at via an experimental procedure that tested different values across all states of the game. The value that gave the best overall performance was $\lambda=0.8$, and thus was selected for all future experiments.

The core feature of any $\mathrm{HC}$ algorithm is the exploration of its neighbourhood. In our specific instantiation, the CGHC, this is achieved by creating a test instance of the game. The value of $T$ determines the numbef of turns used in the test game. In all CGHC experiments the value of $T$ was 100 . Each candidate solution was tested in this fashion. The best solution, according to the fitness function was then used in the "real" game.

The final and most important parameter of any $\mathrm{HC}$ algorithm is the heuristic function, $f$. This is described in section 6.2.1. We mention, though, that the same heuristic function was used in all $\mathrm{HC}, \mathrm{PSO}$, and LA experiments. 


\subsubsection{Particle Swarm Optimization}

Of the many available variations of the canonical PSO algorithm, gbest was used in this work. Once the neighbourhood structure is selected, the question in any PSO algorithm is the number of particles. In this work 30 particles were used in each of the PSO experiments. This number was motivated by the work of Carlisle [15].

Similar to HC, the PSO player was to be able to explore the "terrain" of the game. For this purpose, a testing instance of game was created. The particles traveled though the test instance for a predefined number of turns, $T=100$. Upon completion of the exploration of the test game, the global-best position vector was used to play in the "real" game.

Evaluation of fitness was done using a function, $f$, which is crucial in any PSO . approach. This procedure was implemented in the same manner as with HC and LA algorithms.

\subsection{Learning Automata}

Prior to delving into the specifics of each automaton implementation, it important to discuss the learning problem. The goal of each automaton is to discover the optimal set of probabilities in an instance of the Commons Game. Thus, each automaton is configured with a set of actions $A=\left\{\alpha_{1}, \cdots, \alpha_{5}\right\}$, where $\alpha_{1}$ is the action of selecting the red card, $\alpha_{2}$ is the action of selecting the green card and $\alpha_{3}, \alpha_{4}, \alpha_{5}$ are the actions of selecting yellow, orange and black cards respectively. 


\subsubsection{Environment}

The Commons Game environment was responsible for supplying a response for every received action. The rewards and penalties received by each of the automata are defined as $\beta=\{0,1\}$ where 0 signifies a reward and 1 specifies a penalty. The response received by the automaton is generated in the same fashion without regard the automaton type. At each turn of the game, all players, including the automaton player, submitted their cards selections simultaneously. The environment calculated the number of cards played by grouping them based on color. In the next step the scores for each card group were calculated. The score of the automaton submission was then added to a list of its previous scores. At this stage the penalty/reward response was calculated. A separate procedure is required for response-value calculations due to the fact that game scores range from -20 to 140 .

In every game played, the environment stored a list of scores received by the automaton. The current and previous scores were used to determine whether the response will be a penalty or a reward. At this stage there are a number of possible choices to determine the value of the response. Three approaches were tested, best_ever, best_moving_average and best_current.

- best_ever - Returns a penalty response if the current automaton score is lower than its highest historical score in the current game. Otherwise, a reward is returned.

- best_current - Returns a penalty response if the current automaton score is lower than the highest score of the other players in the current turn. 
- best_moving_average - Returns a penalty response if the current automaton score is lower than the current value of the moving average. The size of the moving average window was set to 10 .

The best automata behavior was achieved via the use of best_moving_average. This fact becomes obvious when we consider the score values generated by the automaton. In the case of best_ever, once a sufficiently high score has been set as a benchmark, every subsequent score value will result in a penalty, even if the game state does not make it possible to achieve a score higher than the benchmark. Therefore, the selection of an optimal action given the current game state will result in a penalty response from the environment. On the other. hand, the best_current criterion does "not apply enough pressure" to the automaton to select the best option. At best the automaton will perform on the level of other (in our case, random selection) players. The best_moving_average does not suffer from the problems of the two approaches above. It constantly pushes the environment towards improving its score.

In addition to the above, none of the previously-described heuristics use any information about the state of the environment. The only available data are the previous rewards.

\subsubsection{Fixed Structure Learning Automata}

Since the Krylov and Krinsky automata are straightforward modifications of the Tsetlin LA, we describe the latter first. It was mentioned previously that Tsetlin devised several different fixed structure designs. The LA used in this work is based 
upon the $L_{2 N, 2}$ automaton. Since the Commons Game presents a problem with five actions, the LA used was the $L_{5 N, N}$ shown in Figure 6.1 where $R, P$ denote reward and penalty transitions.

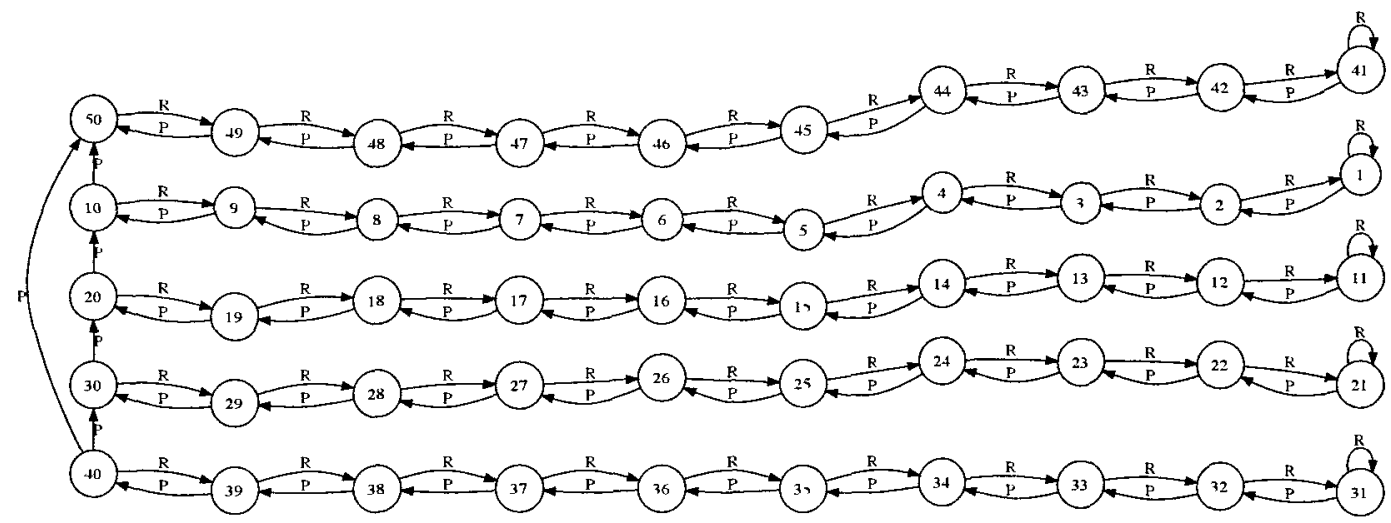

Figure 6.1: Tsetlin's $L_{5 N, N}$ automaton.

The automaton starts in, state $\phi_{\imath 0}$, randomly selected, where $i \in\{1, \cdots, 5\}^{1}$ The action selection is guided is by the automaton's current state. Thus, if the current state is $\phi_{\imath \jmath}$ where $i \in\{1, \cdots, 5\}$ and $j \in\{1, \cdots, 10\}$ the $i^{\text {th }}$ action is selected. For example, if the automaton in a state $\phi_{2 j}$ then the green card is selected. After receiving the penalty or response the automaton transitions from its current state to the next, as can be seen in Figure 6.1.

The Krinsky automaton is a modification of the Tsetlin automaton. This automaton differs in both structure and the way in which transitions are followed. The operation of the $K_{2 N, N}^{1}$ automaton was described in detail in Section 4.2.2.1. In this work we used the $K_{5 N, N}^{1}$, the structure of which can be seen in Figure 6.2 where $R, P$ denote the reward and penalty transitions. Here, same as in $L_{5 N, N}$, if the automaton

\footnotetext{
${ }^{1}$ i.e., the automaton starts in one of the states $\{10,20,30,40,50\}$.
} 
is in a state $\phi_{i j}$, where $i \in\{1, \cdots, 5\}$ and $j \in\{1, \cdots, 10\}$, it selects the $i^{\text {th }}$ action.

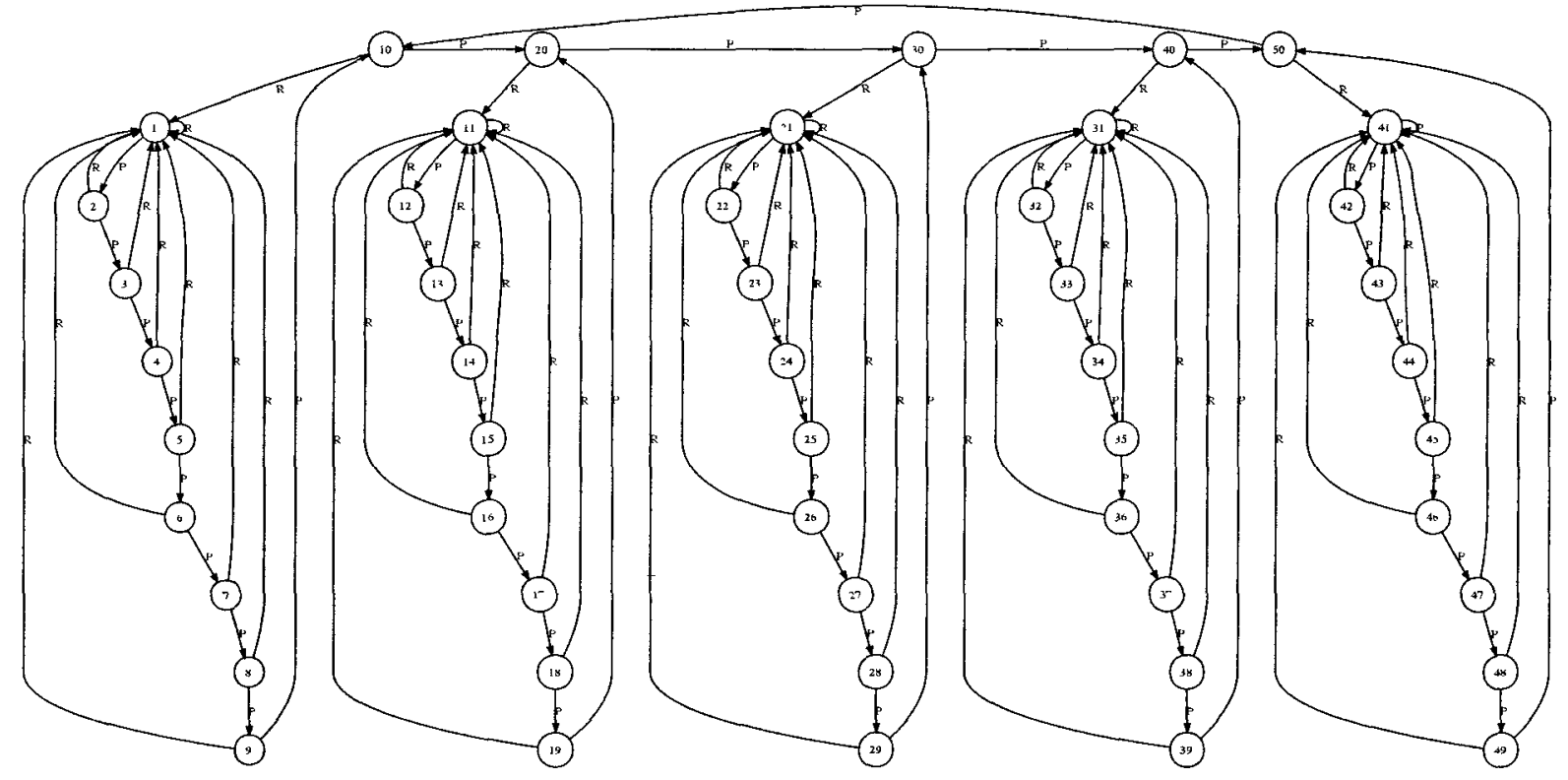

Figure 6.2: Krinsky $K_{5 N, N}^{1}$ automaton.

The Krylov automaton is yet another modification of $L_{2 N, N}$. This automaton, as $K_{2 N, N}^{1}$, differs from $L_{2 N, N}$ in its transitions. The detailed operation and properties of the this LA were described in Section 4.2.2.1. The structure of this automaton is the same the that of the Tsetlin automaton. Similar to $L_{5 N, N}$ and $K_{5 N, N}^{1}$, if the current state is $\phi_{\imath \jmath}$ where $i \in\{1, \cdots, 5\}$ and $j \in\{1, \cdots, 10\}$ the $i^{\text {th }}$ action is selected.

\subsubsection{Variable Structure Learning Automata}

Three types of variable structure automata, the $L_{R I}, L_{R P}$ and $L_{I P}$ were used to play the Commons Game. The precise behavior of these automata is given in Section 4.2.2.2. The remainder of this section will cover implementation specifics only. 
Most of the features of the automata mentioned above are identical. All three use the same number of actions, five. The initial probabilities are initialized as shown below:

$$
P(0)=\left[p_{1}(0), p_{2}(0), \cdots, p_{5}(0)\right] \text { where } p_{\imath}(0)=0.2 \text {. }
$$

The first action in any of the VSLA is selected randomly. Subsequent actions are selected based on the automaton's state and the environment's response. The same environment configuration in used with VSLA as with FSLA.

Unlike FSLA, VSLA use a learning rate parameter, $\lambda$, in the probability updating process. For the purposes of this work, $\lambda=0.95$. In other experimental settings this value was made to vary between $[0.7, \cdots, 0.99]$ depending on the problem. A lower value of $\lambda$ forces a higher rate of change in the probabilities of the LA's actions. At the same time, a higher value of $\lambda$ will have less effect on the action probabilities. The choice of $\lambda=0.95$ was determined empirically. In the context of the Commons Game, fast action switching (which would happen if the value of $\lambda$ was lower) is not desirable as it would lead to a premature convergence. At the start of a game, all the action probabilities are the same. Any change to the values will sway the automaton from one action to the next. In positive states, as well as the lower ones, one of the actions is generally better than the rest. Thus, as the environment slowly oscillates between positive and negative states, the automaton remains capable of maintaining possibility of converging towards the optimal action. 


\subsubsection{Pursuit Automata}

The theoretical details and design of the pursuit automaton were discussed in Section 4.2.3.3. The following paragraphs cover its various parameters and implementation details.

The pursuit automaton uses a learning rate parameter in its probability updating procedures.In this work, the learning rate parameter was set to $\lambda=0.95$. The motivation for specific value is the same as that for VSLA. The remaining variables, $W, Z, D$ are computed either over the course of the interaction with the environment, or upon initialization.

The rest of the details of this automaton were the same as those of VSSA with with the exception of the added steps which updated the vectors $W, Z$ and $D$.

\subsubsection{Estimator Automata}

The configuration of the TS estimator automaton consists of setting a "good" value for the learning parameter, $\lambda$, and selecting the function, $f$. In this case the value $\lambda=0.95$ was used. The suggested family of functions to be used for $f$ for the TSE algorithm is $f(x)=x^{n}$, where $n$ is of the form $1 / k$ or $k$. In this work we used $f(x)=x^{3}$, as suggested by previous work [63].

The TSE algorithm maintains an estimate vector, $D$. Upon initialization each $d_{i}$ is set to a value by selecting each action a pre-set number of times. In this work, the process of initializing $D$ consisted of picking each of the game cards for 100 iterations. 


\section{Chapter 7}

\section{Experimental Results}

The following sections discuss the strategy generation results using the methods described above. For each "intelligent" player a multiple sets of experiments were performed. Each player played 100 games in 6 state range configurations of the game: $[8,-8],[8,8],[8,4],[4,0],[0,-4],[-4,-8]$. Our goal was to determine which of the automata and $\mathrm{HC}$ and PSO generated the best strategy over the 100 games of 1,000 turns in a given state range. Therefore, the results are grouped by the corresponding state ranges.

\subsection{State Range $[8,-8]$}

The $[8,-8]$ state range represents the original configuration of the game as used by Powers [56] in his work. The automata and the random-strategy players are free to direct the environment in whichever direction they please. The results, Table 7.1, 


\begin{tabular}{|c|c|c|c|c|c|c|c|c|}
\hline LA & \% Red & \% Green & \% Yellow & \% Orange & \% Black & Wins & Losses & Avg Score \\
\hline \hline Tsetlin & 0.95237 & 0.00055 & 0.00528 & 0.00081 & 0.04099 & 96 & 4 & 81254.2345 \\
\hline Krinsky & 0.83725 & 0.05152 & 0.00938 & 0.00095 & 0.10090 & 85 & 15 & 70502.9437 \\
\hline Krylov & 0.45613 & 0.14061 & 0.05067 & 0.14287 & 0.20972 & 47 & 53 & 37230.1781 \\
\hline$L_{R I}$ & 1.00000 & 0.00000 & 0.00000 & 0.00000 & 0.00000 & 100 & 0 & 81452.2507 \\
\hline$L_{I P}$ & 0.57830 & 0.11831 & 0.10190 & 0.09969 & 0.10179 & 100 & 0 & 41416.3229 \\
\hline$L_{R P}$ & 0.84177 & 0.05376 & 0.03472 & 0.03512 & 0.03464 & 100 & 0 & 67360.1845 \\
\hline Estimator & 0.91085 & 0.00041 & 0.14182 & 0.00099 & 0.00207 & 94 & 5 & 73680.4199 \\
\hline Pursuit & 0.65000 & 0.04000 & 0.30000 & 0.01000 & 0.00000 & 65 & 35 & 55482.1202 \\
\hline HC & 0.84700 & 0.00400 & 0.03500 & 0.09500 & 0.01900 & 100 & 0 & 57810.4000 \\
\hline PSO & 0.34600 & 0.32900 & 0.12700 & 0.10700 & 0.09100 & 100 & 0 & 11774.2000 \\
\hline
\end{tabular}

Table 7.1: Results of the various algorithms for the state range $[8,-8]$

show that it is, indeed, possible to arrive at the optimal strategy in a complex game, such as the Commons Game, by the use of LA algorithms.

The strategy selected by the $L_{R I}$ automaton is the optimal one for use against random-selection opponents. From Table 7.2 it can be seen that during all the trials the games took place in positive states. Earlier, it was shown that the optimal strategy for this range of states is the red card. The $L_{R I}$ automaton won all the trials that it participated in by. This lends further credibility to the optimality of this strategy. The other VSLA also won all of their games, however, not nearly with as much success as the $L_{R I}$. $L_{I P}$ and $L_{R P}$ used non-optimal strategies by increasing the probability of the green action. As was discussed before, this is a poor choice as there is the probability of a black card being selected by one of the other players is $1 / 5$.

The next best strategy was selected by the Tsetlin automaton. Similar to VSLA, all of the trials for this automaton took place in positive states. Therefore, the expected result is the convergence to unity for the red card action. It did, however, reach an action probability value of 0.95 . In normal circumstances, such a result would be considered a success. Perhaps the most interesting feature of this result is 


\begin{tabular}{|c|c|c|c|c|c|c|c|c|c|c|}
\hline State & Tsetlin & Krinsky & Krylov & $L_{R I}$ & $L_{I P}$ & $L_{R P}$ & Estimator & Pursuit & HC & PSO \\
\hline \hline 8 & 0.0037 & 0.0047 & 0.0000 & 0.0019 & 0.0000 & 0.0000 & 0.0000 & 0.0000 & 0.0000 & 0.0000 \\
\hline 7 & 0.0051 & 0.0048 & 0.0040 & 0.0033 & 0.0003 & 0.0012 & 0.0018 & 0.0066 & 0.0000 & 0.0000 \\
\hline 6 & 0.0409 & 0.0296 & 0.0303 & 0.0385 & 0.0014 & 0.0089 & 0.0447 & 0.0226 & 0.0000 & 0.0000 \\
\hline 5 & 0.2279 & 0.2103 & 0.2075 & 0.2165 & 0.0399 & 0.1121 & 0.2679 & 0.2260 & 0.0000 & 0.0000 \\
\hline 4 & 0.2596 & 0.2311 & 0.2185 & 0.2320 & 0.1095 & 0.1859 & 0.2930 & 0.2519 & 0.0000 & 0.0000 \\
\hline 3 & 0.2549 & 0.2495 & 0.2173 & 0.2591 & 0.2715 & 0.3185 & 0.3042 & 0.2447 & 0.0000 & 0.0000 \\
\hline 2 & 0.1041 & 0.1030 & 0.0939 & 0.1121 & 0.2124 & 0.1678 & 0.1599 & 0.0927 & 0.0000 & 0.0000 \\
\hline 1 & 0.0732 & 0.0784 & 0.0661 & 0.0826 & 0.2044 & 0.1203 & 0.1232 & 0.0795 & 0.4290 & 0.0000 \\
\hline 0 & 0.0307 & 0.0484 & 0.0534 & 0.0539 & 0.1595 & 0.0852 & 0.1423 & 0.0425 & 0.5550 & 0.6640 \\
\hline-1 & 0.0000 & 0.0021 & 0.0042 & 0.0001 & 0.0010 & 0.0002 & 0.0096 & 0.0007 & 00160 & 0.0410 \\
\hline-2 & 0.0000 & 0.0012 & 0.0018 & 0.0000 & 0.0000 & 0.0000 & 0.0073 & 0.0004 & 0.0000 & 0.0200 \\
\hline-3 & 0.0000 & 0.0020 & 0.0023 & 0.0000 & 0.0000 & 0.0000 & 0.0171 & 0.0004 & 0.0000 & 0.0090 \\
\hline-4 & 0.0000 & 0.0014 & 0.0019 & 0.0000 & 0.0000 & 0.0000 & 0.0073 & 0.0005 & 0.0000 & 0.0120 \\
\hline-5 & 0.0000 & 0.0006 & 0.0014 & 0.0000 & 0.0000 & 0.0000 & 0.0024 & 0.0004 & 0.0000 & 0.0270 \\
\hline-6 & 0.0000 & 0.0005 & 0.0012 & 0.0000 & 0.0000 & 0.0000 & 0.0029 & 0.0004 & 0.0000 & 0.0110 \\
\hline-7 & 0.0000 & 0.0082 & 0.0192 & 0.0000 & 0.0000 & 0.0000 & 0.0624 & 0.0071 & 0.0000 & 0.0180 \\
\hline-8 & 0.0000 & 0.0241 & 0.0768 & 0.0000 & 0.0000 & 0.0000 & 0.0391 & 0.0257 & 0.0000 & 0.0980 \\
\hline
\end{tabular}

Table 7.2: Percentage of time spent in the state range $[8,-8]$

that it is the only good result generated by finite structure learning automata.

The Krinsky and Krylov automata failed to find the best action. This can be attributed to the transition configuration of these two automata. The Krinsky automaton moves away from the terminal state on receiving a negative response. It moves, drastically, to the terminal state if the response is positive. Therefore, if the first randomly-selected action, is rewarded it will require 10 consecutive penalties for the machine to move to a different action. In other words, the same behavior that improves convergence speed in other environments has a negative impact here.

The Krylov automaton uses probabilistic transitions for negative responses from the environment. On positive responses, it uses the same strategy as the Tsetlin automaton. As can be seen from the above, this results in the machine sampling all of the actions. While the exploration of the actions may be advantageous in other environments, it is not true in the case of the Commons Game. When the 
states switch, the amount of reward changes. If the automaton is still exploring and has not reached convergence, it will continue exploring. This is also partly due to the environment's response strategy. Since it takes into account the automaton's previous scores, when the environment switches from a higher state to a lower one the same, optimal, action will result in a penalty response. This behavior can slow down the convergence of the Krylov automaton.

The Pursuit automaton failed to find the optimal action given its game state history. The pyrsued action depends on the reward estimate of that actions. The sum of the probabilities of the red and yellow actions is approximately 0.95 . This suggest that the automaton was on the right track in finding the optimal actions.

The Estimator algorithm, given its historical state distribution, performed on par with the finite structure automata. Of the 0.15 probability weight in the yellow card, 0.1 can be attributed to the 10 games played in the states lower than -3 . While the remaining weight of 0.05 is where the it incorrectly estimated the value of the red and yellow actions.

Of the two other computational methods $\mathrm{HC}$ performed significantly better than PSO. The most interesting feature of these results is the insistence of the PSO algorithm on selecting the green card. This behavior helps to keep the environment in one of the negative states. As one can observe, this is not the case with all the other algorithms used. The fact that they avoid playing green, helps to improve the environment as well as their scores. 


\subsection{State Range $[8,8]$}

In the $[8,8]$ state range, all the players received the highest possible available rewards. The best performing LA, as shown in Table 7.3, in this state range was the $L_{R I}$ which selected the first action, the red card, all the time. Several points should be noted here. First of all, only the $L_{R I}$ and the $L_{R P}$ won all of the trials that they participated in. However, due to the fact that $L_{R I}$ picked red all the time, unlike the $L_{R P}$ it scored significantly higher. Secondly, the higher action-probability value of the red card determines the position of the automaton in the overall score rankings. At the same time, out of all the automata only the $L_{R I}$ selected the optimal card.

\begin{tabular}{|c|c|c|c|c|c|c|c|c|}
\hline LA & \% Red & \% Green & \% Yellow & \% Orange & \% Black & Wins & Losses & Avg Score \\
\hline Tsetlin & 0.97310 & 0.00098 & 0.00367 & 0.00085 & 0.02140 & 98 & 2 & 95615.0473 \\
\hline Krinsky & $\mathbf{0 . 9 4 4 5 6}$ & $\mathbf{0 . 0 2 6 3 5}$ & 0.00735 & 0.00078 & 0.02096 & 97 & 3 & 93437.5913 \\
\hline Krylov & $\mathbf{0 . 4 4 8 8 3}$ & $\mathbf{0 . 0 8 0 7 4}$ & 0.04047 & 0.20976 & 0.22020 & 48 & 52 & 44924.1395 \\
\hline$L_{R I}$ & 1.00000 & 0.00000 & 0.00000 & 0.00000 & 0.00000 & 100 & 0 & 94518.1911 \\
\hline$L_{P I}$ & 0.63244 & 0.10116 & 0.09064 & 0.08902 & 0.08674 & 100 & 0 & 56351.9865 \\
\hline$L_{R P}$ & $\mathbf{0 . 8 6 2 3 8}$ & 0.04521 & 0.03099 & 0.03070 & 0.03072 & 100 & 0 & 84639.4063 \\
\hline Estimator & 0.98061 & $\mathbf{0 . 0 3 6 3 4}$ & 0.03625 & 0.00111 & 0.00000 & 92 & 8 & 88527.3393 \\
\hline Pursuit & 0.58000 & $\mathbf{0 . 0 0 0 0 1}$ & 0.35998 & 0.05001 & 0.01001 & 58 & 42 & 57481.9410 \\
\hline HC & 0.74300 & 0.09900 & 0.05300 & 0.05200 & 0.05300 & 100 & 0 & 76645.8000 \\
\hline PSO & 0.32400 & 0.33400 & 0.13700 & 0.11000 & 0.09500 & 100 & 0 & 37912.1500 \\
\hline
\end{tabular}

Table 7.3: Results of the various algorithms for the state range $[8,8]$

Of the two other methods, the PSO continually attempted to maximize its score by playing green. However, this aggressiveness was severely punished by the other players. 


\subsection{State Range [8, 4]}

The $[8,4]$ state range represents the upper quartile of the full range of game states. As in $[8,8]$ and $[8,-8]$ the $L_{R I}$ and TSE automata performed best. Also, as in the ranges presented above, the TS Estimator, $L_{R I}$ and Tsetlin automata approached the optimal action probability solution. These results are shown in Table 7.4 and Table 7.5.

\begin{tabular}{|c|c|c|c|c|c|c|c|c|}
\hline LA & \% Red & \% Green & \% Yellow & \% Orange & \% Black & Wins & Losses & Avg Score \\
\hline \hline Tsetlin & 0.96371 & 0.00062 & $\mathbf{0 . 0 0 3 6 8}$ & 0.00095 & 0.03104 & 97 & 3 & 90754.8777 \\
\hline Krinsky & 0.94054 & 0.00139 & 0.00637 & 0.00080 & 0.05090 & 95 & 5 & 88465.9787 \\
\hline Krylov & 0.46615 & 0.08151 & $\mathbf{0 . 0 2 7 9 3}$ & 0.14164 & 0.28277 & 48 & 52 & 44442.1254 \\
\hline$L_{R I}$ & 1.00000 & 0.00000 & 0.00000 & 0.00000 & 0.00000 & 100 & 0 & 90587.7760 \\
\hline$L_{P I}$ & 0.62807 & 0.10061 & 0.09021 & $\mathbf{0 . 0 9 1 3 7}$ & 0.08974 & 100 & 0 & $\mathbf{5 3 3 9 4 . 5 2 6 6}$ \\
\hline$L_{R P}$ & 0.86271 & 0.04290 & $\mathbf{0 . 0 3 1 7 1}$ & 0.03126 & 0.03143 & 100 & 0 & 79871.8438 \\
\hline Estimator & 0.91782 & 0.03500 & $\mathbf{0 . 0 7 9 9 4}$ & $\mathbf{0 . 0 2 2 6 0}$ & 0.00125 & 85 & 14 & $\mathbf{7 7 9 0 2 . 2 8 1 4}$ \\
\hline Pursuit & 0.51000 & 0.00000 & $\mathbf{0 . 4 2 0 0 0}$ & $\mathbf{0 . 0 4 0 0 0}$ & 0.03000 & $\mathbf{5 1}$ & 49 & 49377.2861 \\
\hline HC & 0.70000 & 0.12900 & $\mathbf{0 . 0 5 0 0 0}$ & $\mathbf{0 . 0 6 2 0 0}$ & 0.05900 & 100 & 0 & 67920.4000 \\
\hline PSO & 0.32000 & 0.34000 & $\mathbf{0 . 1 2 3 0 0}$ & $\mathbf{0 . 1 2 3 0 0}$ & 0.09400 & 100 & 0 & 35472.9500 \\
\hline
\end{tabular}

Table 7.4: Results of the various algorithms for the state range $[8,4]$

\begin{tabular}{|c|c|c|c|c|c|}
\hline LA & 8 & 7 & 6 & 5 & 4 \\
\hline \hline Tsetlin & 0.0000 & 0.0072 & 0.0616 & 0.6566 & 0.2745 \\
\hline Krinsky & 0.0000 & 0.0031 & 0.0690 & 0.6547 & 0.2732 \\
\hline Krylov & 0.0000 & 0.0075 & 0.0568 & 0.6262 & 0.3095 \\
\hline$L_{r \imath}$ & 0.0000 & 0.0044 & 0.0534 & 0.6615 & 0.2807 \\
\hline$L_{p \imath}$ & 0.0000 & 0.0015 & 0.0307 & 0.6201 & 0.3478 \\
\hline$L_{r p}$ & 0.0098 & 0.0036 & 0.0420 & 0.6391 & 0.3054 \\
\hline Estimator & 0.0000 & 0.0071 & 0.0678 & 0.9241 & 0.4860 \\
\hline Pursuit & 0.0000 & 0.0036 & 0.0571 & 0.6526 & 0.2867 \\
\hline HC & 0.0000 & 0.0000 & 0.0200 & 0.5520 & 0.4280 \\
\hline PSO & 0.0000 & 0.0000 & 0.0190 & 0.4420 & 0.5390 \\
\hline
\end{tabular}

Table 7.5: Percentage of time spent in a given state for state range $[8,4]$ 


\subsection{State Range $[4,0]$}

The results in the $[4,0]$ range are nearly identical the results generated in the $[8,-8]$, $[8,4]$ ranges. The optimal action in this case was playing red. These results are shown in Tables 7.6 and 7.7 .

\begin{tabular}{|c|c|c|c|c|c|c|c|c|}
\hline LA & \% Red & \% Green & \% Yellow & \% Orange & \% Black & Wins & Losses & Avg Score \\
\hline \hline Tsetlin & 0.97276 & 0.00111 & 0.00365 & 0.00110 & 0.02138 & 98 & 2 & 74554.3367 \\
\hline Krinsky & 0.87887 & 0.02286 & 0.00667 & 0.00079 & 0.09081 & 90 & 10 & 67330.4650 \\
\hline Krylov & 0.37531 & 0.07130 & 0.03798 & 0.19913 & 0.31628 & 39 & 61 & 27620.1528 \\
\hline$L_{R I}$ & 1.00000 & 0.00000 & 0.00000 & 0.00000 & 0.00000 & 100 & 0 & 71512.4740 \\
\hline$L_{P I}$ & 0.57784 & 0.11562 & 0.10225 & 0.10099 & 0.10330 & 100 & 0 & 34069.7613 \\
\hline$L_{R P}$ & 0.83185 & 0.05281 & 0.03814 & 0.03844 & 0.03876 & 100 & 0 & 58841.4873 \\
\hline Estimator & 0.95838 & 0.01349 & 0.08224 & 0.00011 & 0.00033 & 90 & 9 & 67340.6330 \\
\hline Pursuit & 0.53000 & 0.06000 & 0.38000 & 0.02000 & 0.01000 & 53 & 47 & 42026.9131 \\
\hline HC & 0.53900 & 0.13100 & 0.12300 & 0.10900 & 0.09800 & 100 & 0 & 38604.5667 \\
\hline PSO & 0.34000 & 0.32900 & 0.12200 & 0.11900 & 0.09000 & 100 & 0 & 20525.3571 \\
\hline
\end{tabular}

Table 7.6: Results of the various algorithms for the state range $[4,0]$

\begin{tabular}{|c|c|c|c|c|c|}
\hline LA & 4 & 3 & 2 & 1 & 0 \\
\hline \hline Tsetlin & 0.2970 & 0.1500 & 0.2207 & 0.2575 & $\mathbf{0 . 0 7 4 8}$ \\
\hline Krinsky & $\mathbf{0 . 2 8 4 4}$ & $\mathbf{0 . 1 5 9 9}$ & 0.2273 & 0.2484 & $\mathbf{0 . 0 8 0 0}$ \\
\hline Krylov & 0.2459 & 0.1460 & 0.2155 & 0.2794 & 0.1132 \\
\hline Lri & 0.2350 & $\mathbf{0 . 1 5 0 4}$ & 0.2293 & 0.2979 & $\mathbf{0 . 0 8 7 5}$ \\
\hline Lpi & $\mathbf{0 . 0 3 0 7}$ & $\mathbf{0 . 0 4 9 1}$ & 0.1954 & 0.5149 & $\mathbf{0 . 2 0 9 9}$ \\
\hline Lrp & 0.1773 & $\mathbf{0 . 0 9 5 1}$ & 0.2310 & 0.3683 & $\mathbf{0 . 1 2 8 2}$ \\
\hline Estimator & 0.3345 & $\mathbf{0 . 1 7 2 0}$ & 0.3231 & 0.4690 & $\mathbf{0 . 1 8 6 5}$ \\
\hline Pursuit & $\mathbf{0 . 2 8 3 7}$ & $\mathbf{0 . 1 2 4 5}$ & 0.2226 & 0.2667 & $\mathbf{0 . 1 0 2 4}$ \\
\hline HC & $\mathbf{0 . 0 0 0 0}$ & $\mathbf{0 . 1 8 0 0}$ & 0.2090 & 0.4260 & $\mathbf{0 . 1 8 5 0}$ \\
\hline PSO & $\mathbf{0 . 0 0 0 0}$ & $\mathbf{0 . 0 0 0 0}$ & 0.0270 & 0.4920 & $\mathbf{0 . 4 8 1 0}$ \\
\hline
\end{tabular}

Table 7.7: Percentage of time spent in a given state for the state range $[4,0]$

\subsection{State Range [0, -4]}

In the $[0,-4]$ state range, the players were forced to contend in a somewhat degraded environment. The trend established in the environments presented earlier continued 
here, as shown in Tables 7.8 and 7.9. The $L_{R I}$ automaton won all of the trials by exclusively selecting the cooperative action. This direction is consistent with the computational solution for the Commons Game, as cooperation is the best action for this state range.

\begin{tabular}{|c|c|c|c|c|c|c|c|c|}
\hline LA & \% Red & \% Green & \% Yellow & \% Orange & \% Black & Wins & Losses & Avg Score \\
\hline \hline Tsetlin & 0.96576 & 0.00130 & 0.01044 & 0.00104 & 0.02146 & 98 & 2 & 29972.1956 \\
\hline Krinsky & 0.86232 & 0.02455 & 0.01139 & 0.00089 & 0.10085 & 88 & 12 & 22404.8934 \\
\hline Krylov & 0.42973 & 0.08035 & 0.08546 & 0.22097 & 0.18349 & 51 & 49 & 11681.7099 \\
\hline$L_{R I}$ & 0.99001 & 0.00001 & 0.00995 & 0.00003 & 0.00000 & 100 & 0 & 25638.9866 \\
\hline$L_{P I}$ & 0.37638 & 0.12754 & 0.26243 & 0.12004 & 0.11361 & 100 & 0 & 6530.7039 \\
\hline$L_{R P}$ & 0.71209 & 0.07362 & 0.11159 & 0.05145 & 0.05125 & 100 & 0 & 15144.3326 \\
\hline Estimator & 0.84411 & 0.02009 & 0.17930 & 0.01145 & 0.00083 & 92 & 7 & 25703.6486 \\
\hline Pursuit & 0.48002 & 0.00000 & 0.43998 & 0.06000 & 0.02000 & 72 & 28 & 16908.6615 \\
\hline HC & 0.42100 & 0.08500 & 0.21400 & 0.12600 & 0.15400 & 100 & 0 & 7306.8333 \\
\hline PSO & 0.35400 & 0.33300 & 0.12900 & 0.10200 & 0.08200 & 23 & 77 & -1530.6000 \\
\hline
\end{tabular}

Table 7.8: Results of the various algorithms for the state range $[0,-4]$

\begin{tabular}{|c|c|c|c|c|c|}
\hline LA & 0 & -1 & -2 & -3 & -4 \\
\hline \hline Tsetlin & 0.4689 & 0.0723 & 0.1042 & 0.2624 & 0.0921 \\
\hline Krinsky & 0.3728 & 0.0721 & 0.1076 & 0.3204 & 0.1271 \\
\hline Krylov & 0.4737 & 0.0585 & 0.0853 & 0.2453 & 0.1372 \\
\hline Lri & 0.3776 & 0.0568 & 0.1066 & 0.3424 & 0.1167 \\
\hline Lpi & 0.1542 & 0.0283 & 0.0779 & 0.4857 & 0.2539 \\
\hline Lrp & 0.2217 & 0.0429 & 0.1019 & 0.4488 & 0.1848 \\
\hline Estimator & 0.5941 & 0.0632 & 0.1195 & 0.4793 & 0.2289 \\
\hline Pursuit & 0.4227 & 0.0597 & 0.1202 & 0.2951 & 0.1023 \\
\hline HC & 0.0590 & 0.0640 & 0.2780 & 0.4610 & 0.1380 \\
\hline PSO & 0.0000 & 0.0000 & 0.0240 & 0.4170 & 0.5590 \\
\hline
\end{tabular}

Table 7.9: Percentage of time spent in a given state for the state range $[0,-4]$

\subsection{State Range $[-4,-8]$}

$[-4,-8]$ is the final state range used to conduct a competitions between the HC, PSO, LA algorithms and random players. The optimal action for this range is, complete 


\begin{tabular}{|c|c|c|c|c|c|c|c|c|}
\hline LA & \% Red & \% Green & \% Yellow & \% Orange & \% Black & Wins & Losses & Avg Score \\
\hline \hline Tsetlin & 0.45130 & 0.02726 & 0.43921 & 0.02103 & 0.06120 & 96 & 4 & 1385.4777 \\
\hline Krinsky & 0.51050 & 0.21466 & 0.20063 & 0.00706 & 0.06715 & 67 & 33 & -2979.0269 \\
\hline Krylov & 0.26501 & 0.13712 & 0.12606 & 0.21487 & 0.25694 & 56 & 44 & -3312.4293 \\
\hline$L_{R I}$ & 0.00109 & 0.00038 & 0.99780 & 0.00040 & 0.00033 & 100 & 0 & 5407.9600 \\
\hline$L_{P I}$ & 0.09460 & 0.08782 & 0.65223 & 0.08236 & 0.08300 & 100 & 0 & 380.5881 \\
\hline$L_{R P}$ & 0.05165 & 0.04660 & 0.81649 & 0.04274 & 0.04252 & 100 & 0 & 3578.0589 \\
\hline Estimator & 0.08003 & 0.00128 & 0.91892 & 0.03637 & 0.01406 & 100 & 0 & 4977.6364 \\
\hline Pursuit & 0.03000 & 0.00000 & 0.93000 & 0.03000 & 0.01000 & 96 & 4 & 5352.2998 \\
\hline HC & 0.03000 & 0.00400 & 0.95100 & 0.00600 & 0.00900 & 100 & 0 & 5621.6667 \\
\hline PSO & 0.13000 & 0.04900 & 0.62600 & 0.10400 & 0.09100 & 100 & 0 & 2128.2071 \\
\hline
\end{tabular}

Table 7.10: Results of the various algorithms for the state range $[-4,-8]$

\begin{tabular}{|c|c|c|c|c|c|}
\hline LA & -4 & -5 & -6 & -7 & -8 \\
\hline \hline Tsetlin & 0.0000 & 0.0058 & 0.0305 & 0.4728 & 0.4908 \\
\hline Krinsky & 0.0000 & 0.0066 & 0.0320 & 0.4144 & 0.5471 \\
\hline Krylov & 0.0195 & 0.0057 & 0.0329 & 0.4345 & 0.5074 \\
\hline Lri & 0.0000 & 0.0040 & 0.0264 & 0.4862 & 0.4834 \\
\hline Lpi & 0.0000 & 0.0029 & 0.0223 & 0.4472 & 0.5276 \\
\hline Lrp & 0.0099 & 0.0042 & 0.0271 & 0.4688 & 0.4900 \\
\hline Estimator & 0.0148 & 0.0112 & 0.0320 & 0.6950 & 0.7471 \\
\hline Pursuit & 0.0000 & 0.0079 & 0.0338 & 0.4824 & 0.4759 \\
\hline HC & 0.0000 & 0.0110 & 0.0660 & 0.5490 & 0.3740 \\
\hline PSO & 0.0000 & 0.0000 & 0.0150 & 0.2840 & 0.7010 \\
\hline
\end{tabular}

Table 7.11: Percentage of time spent in a given state for the state range $[-4,-8]$

abstention, the yellow card. Here none of the algorithms converged to unity, as can be seen in Tables 7.10 and 7.11. The $L_{R I}$ automaton was the closest with a value of 0.9967 of probability in the yellow action. Equally interesting is the action probability distribution of the Tsetlin automaton, which in other state ranges placed the most probability weight into the correct action. However, in this range, it selected red with a probability of approximately 0.45 . 


\subsection{Results in Games where $N>8$}

The discussion above focused on games where the number of players is set to 8 . Experiments conducted with the $L_{R I}$ automaton in games where $N \in\{15,30\}$ produce the same optimal strategies, as shown in Tables 7.12 and 7.13. This provides further confirmation of the claims regarding best strategies for games with random action-selection opponents.

\begin{tabular}{|c|c|c|c|c|c|c|c|c|}
\hline State Range & \% Red & \% Green & \% Yellow & \% Orange & \% Black & Wins & Losses & Avg Score \\
\hline \hline$[8,-8]$ & 0.07181 & 0.00012 & 0.92778 & 0.00018 & 0.00012 & 100 & 0 & 5352.9956 \\
\hline$[8,8]$ & 1.00000 & 0.00000 & 0.00000 & 0.00000 & 0.00000 & 100 & 0 & 62872.4121 \\
\hline$[8,4]$ & 1.00000 & 0.00000 & 0.00000 & 0.00000 & 0.00000 & 100 & 0 & 58601.7706 \\
\hline$[4,0]$ & 1.00000 & 0.00000 & 0.00000 & 0.00000 & 0.00000 & 100 & 0 & 33694.3297 \\
\hline$[0,-4]$ & 0.12485 & 0.00001 & 0.87511 & 0.00002 & 0.00001 & 100 & 0 & 5727.6645 \\
\hline$[-4,-8]$ & 0.03113 & 0.00019 & 0.96791 & 0.00050 & 0.00027 & 100 & 0 & 5212.4997 \\
\hline
\end{tabular}

Table 7.12: $L_{R I}$ generated strategies for use in games with 15 players

\begin{tabular}{|c|c|c|c|c|c|c|c|c|}
\hline State Range & \% Red & \% Green & \% Yellow & \% Orange & \% Black & Wins & Losses & Avg Score \\
\hline \hline$[8,-8]$ & 0.01124 & 0.00020 & 0.98776 & 0.00054 & 0.00026 & 100 & 0 & 5401.3704 \\
\hline$[8,8]$ & 0.99997 & 0.00000 & 0.00002 & 0.00001 & 0.00000 & 100 & 0 & 28836.2675 \\
\hline$[8,4]$ & 1.00000 & 0.00000 & 0.00000 & 0.00000 & 0.00000 & 100 & 0 & 26963.5022 \\
\hline$[4,0]$ & 1.00000 & 0.00000 & 0.00000 & 0.00000 & 0.00000 & 100 & 0 & 16021.5810 \\
\hline$[0,-4]$ & 0.00317 & 0.00007 & 0.99655 & 0.00012 & 0.00008 & 100 & 0 & 5601.2667 \\
\hline$[-4,-8]$ & 0.00115 & 0.00018 & 0.99780 & 0.00062 & 0.00024 & 100 & 0 & 5407.8276 \\
\hline
\end{tabular}

Table 7.13: $L_{R I}$ generated strategies for use in games with 30 players

The overall summary of the obtained results is given in the next chapter. 


\section{Chapter 8}

\section{Conclusion}

\subsection{Summary}

This thesis presented the first results which concern the application of learning strategies to the Commons Game, a complex N-player social dilemma game. Chapter 2 gave an overview of several games that have previously been mathematically analyzed. Chapter 3 provided a full description of the properties of the Commons Game. In the same chapter, the first computational results in relation to the game were presented. These results showed that the intuitive strategy of alternating between red and green cards does not yield optimal results if random action-section opponents participate in the game. The optimal strategy, in this case, is for the participant to play cooperatively, red, as long as the environment is not completely degraded (states $[8, \cdots,-3]$ ). If the state of the environment is poor (states $[-4, \cdots,-8]$ ) the strategy requires a switch to abstention, the yellow card. 
With the first results in hand, a review of learning automata and their properties was given in Chapter 4. Continuing on, Chapter 5 described several different versions of the hill-climbing algorithm, as well as presented a new modification of the same algorithm. The modifications facilitated being able to apply hill-climbing to the problem of generating "good" strategies for the Commons Game when faced with random action-selection opponents. A description of the particle swarm optimization algorithm was also given in this chapter. Chapter 6 described the method used and experimental settings.

Chapter 7 explored the results of application of the algorithms mentioned above. The results show that LA can converge to the optimal action even in the presence of difficult opponents. In short, the $L_{R I}$ automaton always converged, with unit probability, to the optimal (as determined in Chapter 2) action and consistently won all of the games it participated in. Good results (i.e., convergence in $\approx 95 / 100$ of games) were also obtained by the use of the TS estimator algorithm. Somewhat surprisingly, the Tsetlin automaton also converged to the optimal action for a vast majority of trials (i.e., close to $95 \%$ ). The experimental results generated by the $\mathrm{HC}$ and PSO in some cases approach those of the LA. However, in the majority of cases they fell short. This result is not overly surprising, as it is well known that both the PSO and HC have a tendency to get "stuck" in local optima.

Given the observed results the following conclusions can be made. In an environment for which the properties have not been studied in depth by formal mathematical analysis, one can successfully apply LA algorithms to obtain near-optimal solutions. Of the automata families, the less computationally complex, variable structure 
schemes should be used first. They require little parameter adjustment and computational power. The finite structure and estimator algorithms can also be successfully used. However, this can be avoided if VSSA have already proven favourable. The results also showed that although $\mathrm{HC}$ and PSO perform better than random-selection participants, they are not likely to converge to the optimal action. The running time of these two algorithms is also considerably larger than that of any LA scheme. Thus, it is difficult to recommend the use of HC or PSO in environments such as the Commons Game.

\subsection{Future Work}

The analysis presented in this work opens several new directions for research. In some cases the random-selection opponents drive the environment into negative states. The question of whether the same would occur if the random-selection opponents were to be replaced with a different action-selection strategy remain open. Another question that is unanswered is the following: What would happen if the LAs were augmented with additional information about its opponents. Approaches in pattern recognition suggest that such LA can be designed. We also note that all the games played in this research were conducted with 8 players. However, the Commons Game can be played by up to 50 players. Large groups of players would enable formation of coalitions. By allowing communication between the coalition members, it may be possible to design strategies that further increase the returns of a single member of the group while ensuring that no other player in the game receives a higher reward. 


\section{Bibliography}

[1] A. M. Abdelbar, S. Ragab, and S. Mitri. Applying Co-Evolutionary Particle Swam Optimization to the Egyptian Board Game Seega. In Proceedings of The First Asian-Pacific Workshop on Genetic Programming, pages 9-15, 2003.

[2] M. Agache. Families of Estimator-Based Schochastic Learning Algorithms. Master's thesis, Carleton University, 2000.

[3] M. Agache and B.J. Oommen. Generalized Pursuit Learning Schemes: New Families of Continuous and Discretized Learning Automata. Systems, Man, and Cybernetics, Part B: Cybernetics, IEEE Transactions on, 32(6):738-749, 2002.

[4] Vladimir Akimov and Mikhail Soutchanski. Automata Simulation of N-Person Social Dilemma Games. The Journal of Conflict Resolution, 38(1):pp. 138-148, 1994.

[5] R.C. Atkinson, G.H. Bower, and E.J. Crothers. Introduction to Mathematical Learning Theory. Wiley, 1965.

[6] R. Axelrod. Evolving New Strategies. Genetic Algorithm and Simulated Annealing, London, pages 32-41, 1987. 
[7] N. Baba. The Commons Game by Microcomputer. Simulation 8 Gaming, 15:487-492, 1984.

[8] N. Baba. Utilization of Genetic Algorithms in Order to Make Game Playing Much More Exciting. In Knowledge-Based Intelligent Information Engineering Systems, 1999. Third International Conference, pages 473-476, 1999.

[9] N. Baba and H. Handa. COMMONS GAME Made More Exciting by an Intelligent Utilization of the Two Evolutionary Algorithms. In Advanced Intelligent Paradigms in Computer Games, volume 71 of Studies in Computational Intelligence, pages 1-16. Springer Berlin / Heidelberg, 2007.

[10] N. Baba, K. Nagasawa, and H. Handa. Utilization of Soft Computing Techniques for Making Environmental Games More Exciting -Toward an Effective Utilization of the COMMONS GAME. 5178 LNAI(PART 2):411-417, 2008.

[11] A.G. Barto and P. Anandan. Pattern-recognizing Stochastic Learning Automata. IEEE Transactions on Systems, Man, \& Cybernetics, pages 360-375, 1985.

[12] H. Beigy and M. Meybodi. Call Admission Control in Cellular Mobile Networks: A Learning Automata Approach. EurAsia-ICT 2002: Information and Communication Technology, pages 450-457, 2002.

[13] E.A. Billard. Evolutionary Strategies of Stochastic Learning Automata in the Prisoner's Dilemma. Biosystems, 39(2):93-107, 1996.

[14] R.R. Bush and F. Mosteller. Stochastic models for learning. John Wiley \& Sons, Inc., 1955. 
[15] A. Carlisle and G. Dozier. An Off-the-shelf PSO. In Proceedings of the workshop on particle swarm optimization, volume 1, pages 1-6. Indianapolis, 2001.

[16] M. Clerc. Particle swarm optimization. Wiley-ISTE, 2006.

[17] J. Conradie and A. P. Engelbrecht. Training bao game-playing agents using coevolutionary particle swarm optimization. In Proceedings of the IEEE 2006 Symposium on Computational Intelligence and Games, pages 67-74, 2006.

[18] R. M. Dawes. Social Dilemmas. Annual Review of Psychology, 31(1):169-193, 1980.

[19] R. C. Eberhart, Y. Shi, J. Kennedy, and Ebooks Corporation. Swarm intelligence. Elsevier, 2001.

[20] A. P. Engelbrecht. Fundamentals of computational swarm intelligence, volume 1. Wiley London, 2005.

[21] N. Faysse. Coping with the Tragedy of the Commons: Game Structure and Design of Rules. Journal of Economic Surveys, 19(2):239-261, 2005.

[22] S. Forrest and M. Mitchell. Relative Building-block Fitness and the Buildingblock Hypothesis. Ann Arbor, 1001:48109.

[23] N. Franken and A. P. Engelbrecht. Particle Swarm Optimization Approaches to Coevolve Strategies for the Iterated Prisoner's Dilemma. IEEE Transactions on Evolutionary Computation, 9(6):562-579, 2005.

[24] G. Hardin. The Tragedy of the Commons. Science, 162(3859):1243-1248, 1968. 
[25] G. Hardin. ESSAYS ON SCIENCE AND SOCIETY: Extensions of "The Tragedy of the Commons". Science, 280(5364):682-683, 1998.

[26] G. Hardin and J. Baden. Managing the Commons. Freeman San Francisco, 1977.

[27] F. Heppner and U. Grenander. A Stochastic Nonlinear Model for Coordinated Bird Flocks. American Association for the Advancement of Sience, Washington, DC(USA), 1990.

[28] J. Kabudian, M. R. Meybodi, and M. M. Homayounpour. Applying Continuous Action Reinforcement Learning Automata (CARLA) to Global Training of Hidden Markov Models. In International Conference on Information Technology: Coding and Computing (ITCC '04), volume 4, pages 638 -642. IEEE Computer Society, 2004.

[29] J. Kennedy and R. C. Eberhart. Particle Swarm Optimization. In Proceedings of IEEE International Conference on Neural Networks, volume 4, pages 1942-1948, 1995.

[30] C. A. Kirts, M. A. Tumeo, and J. M. Sinz. The COMMONS GAME: Its Instructional Value When Used in a Natural Resources Management Context. Simulation $\&$ Gaming, 22(1):5-18, 1991.

[31] S. S. Komorita and C. D. Parks. Social dilemmas. Brown \& Benchmark, 1994.

[32] V. I. Krinsky. Asymptotically Optimal Automaton with Exponential Seed of Convergence. Biofizica, 9:484-487, 1964.

[33] V. Y. Krylov. One Stochastic Automaton which is Asymptotically Optimal in Random Medium. Automata Remote Control, 24:1114-1116, 1964. 
[34] J. K. Lanctot and B. J. Oommen. Discretized Estimator Learning Automata. Systems, Man and Cybernetics, IEEE Transactions on, 22(6):1473-1483, 1992.

[35] E. C. Laskari, K. E. Parsopoulos, and M. N. Vrahatis. Particle Swarm Optimization for Minimax Problems. In Proceedings of the IEEE 2002 Congress on Evolutionary Computation, pages 1582-1587, 2002.

[36] U. Lorenz and T. Tscheuschner. Player modeling, search algorithms and strategies in multi player games. In Advances in Computer Games, volume 4250 of Lecture Notes in Computer Science, pages 210-224, 2005.

[37] M. A. Mazurowski and J. M. Zurada. Solving Multi-agent Control Problems Using Particle Swarm Optimization. In IEEE Swarm Intelligence Symposium, 2007. SIS 2007, pages 105-111, 2007.

[38] K. S. Narendra and M. A. L. Thathachar. Learning automata: An Introduction. Prentice-Hall, Inc. Upper Saddle River, NJ, USA, 1989.

[39] O. V. Nedzelnitsky Jr and K. S. Narendra. Nonstationary Models of Learning Automata Routing in Data Communication Networks. IEEE transactions on systems, man, and cybernetics, 17(6):1004-1015, 1987.

[40] M. F. Norman. On the Linear Model with Two Absorbing Barriers. Journal of Mathematical Psychology, 5(2):225-241, 1968.

[41] S. Ohnuma. Environmental Commons Game: Is the Free Rider a "Bad Apple"? In Gaming, Simulations, and Society, pages 21-28. Springer Tokyo, 2005. 
[42] K. O'Neill and S. Pittsburgh. Real-Time Tragedies: A Simulated" Commons Learning Laboratory. Proceedings System Dynamics 1992-Supplement, pages 25-34, 1992.

[43] B. J. Oommen and J. P. R. Christensen. $\epsilon$-optimal Discretized Linear RewardPenalty Learning Automata. Systems, Man and Cybernetics, IEEE Transactions on, 18(3):451-458, 1988.

[44] B. J. Oommen and E. Hansen. The Asymptotic Optimality of Discretized Linear Reward-Inaction Learning Automata. IEEE transactions on systems, man, and cybernetics, 14(3):542-545, 1984.

[45] B. J. Oommen and J. K. Lanctot. 6 -optimal Discretized Pursuit Learning Automata. In Systems, Man and Cybernetics, 1989. Conference Proceedings., IEEE International Conference on, pages $6-12$ vol.1, nov 1989.

[46] B. J. Oommen and S. Misra. Cybernetics and Learning Automata. Springer Handbook of Automation, pages 221-235, 2009.

[47] B.J. Oommen. Graph Partitioning Using Learning Automata. Computers, IEEE Transactions on, 45(2):195-208, 1996.

[48] B.J. Oommen and J.K. Lanctôt. Discretized Pursuit Learning Automata. Systems, Man and Cybernetics, IEEE Transactions on, 20(4):931-938, 1990.

[49] M. J. Osborne and A. Rubinstein. A Course in Game Theory. The MIT press, 1994. 
[50] G. I. Papadimitriou and A. S. Pomportsis. Learning-automata-based TDMA protocols for broadcast communication systems with bursty traffic. Communications Letters, IEEE, 4(3):107-109, 2000.

[51] J. Platt. Social traps. American Psychologist, 28(8):641, 1973.

[52] R. Poli. Analysis of the Publications on the Applications of Particle Swarm Optimisation. Journal of Artificial Evolution and Applications, 2008:3, 2008.

[53] R. Poli, J. Kennedy, and T. Blackwell. Particle Swarm Optimization. Swarm intelligence, 1(1):33-57, 2007.

[54] W. Poundstone. Prisoner's Dilemma. Doubleday, 1992.

[55] R. B. Powers and W. Boyle. Generalization From a Commons-Dilemma Game: The Effects of a Fine Option, Information, and Communication on Cooperation and Defection. Simulation Gaming, 14(3):253-274, 1983.

[56] R. B. Powers, R. E. Duss, and R. S. Norton. THE COMMONS GAME Manual, 1977.

[57] A. Rapoport and A.M. Chammah. The game of chicken. American Behavioral Scientist, 10(3):10, 1966.

[58] W. T. Reeves. Particle Systems: A Technique for Modeling a Class of Fuzzy Objects. ACM Transactions on graphics, 2(2):91-108, 1983.

[59] C. W. Reynolds. Flocks, Herds and Schools: A Distributed Behavioral Model. In ACM SIGGRAPH Computer Graphics, volume 21, pages 25-34. ACM, 1987. 
[60] S. J. Russell, P. Norvig, J. F. Candy, J. M. Malik, and D. D. Edwards. Artificial Intelligence: A Modern Approach. Prentice hall, 2010.

[61] A. L. Samuel. Some Studies in Machine Learning Using the Game of Checkers. IBM J. Res. Dev., 3(3):210-229, 1959.

[62] A. L. Samuel. Some Studies in Machine Learning Using the Game of Checkers. II: Recent Progress. IBM J. Res. Dev., 11(6):601-617, 1967.

[63] P. S. Sastry. Systems of learning automata: Estimator algorithms applications. $\mathrm{PhD}$ thesis, Dept. of Electrical Engineering, Inidian Institute of Science, 1985.

[64] I. J. Shapiro and K. S. Narendra. Use of Stochastic Automata for Parameter Self-optimization with Multimodal Performance Criteria. Systems Science and Cybernetics, IEEE Transactions on, 5(4):352-360, 1969.

[65] B. Skyrms. The Stag Hunt and the Evolution of Social Structure. Cambridge Univ Pr, 2004.

[66] J. A. Stankovic. Stability and Distributed Scheduling Algorithms. IEEE Transactions on Software Engineering, pages 1141-1152, 1985.

[67] N. Sturtevant. A Comparison of Algorithms for Multi-Player Games. In Proceedings of the Third International Conference on Computers and Games, pages $108-122,2002$.

[68] N. Sturtevant. Current Challenges in Multi-player Game Search. In Proceedings of the 4th International Conference on Computers and Games, volume 3846, pages 285-300. Springer Berlin / Heidelberg, 2006. 
[69] M. K. Sudareshan and T. A. Condarcure. Recurrent Neural-Network Training by a Learning Automaton Approach for Trajectory Learning and Control System Design. Neural Networks, IEEE Transactions on, 9(3):354-368, 1998.

[70] R. S. Sutton and A. G. Barto. Reinforcement Learning: An Introduction. MIT Press, 2004.

[71] M. N. Szilagyi. Solutions to Realistic Prisoners' Dilemma Games. In 2001 IEEE International Conference on Systems, Man, and Cybernetics, volume 2, pages 841-846, 2001.

[72] M. N. Szilagyi. Simulation of Multi-Agent Prisoners Dilemmas. Systems Analysis Modelling Simulation, 43:829-846, 2003.

[73] M. A. L Thathachar and B. J. Oommen. Discretized Reward-Inaction Learning Automata. J. Cybern. Inf. Sci, 2(1):24-29, 1979.

[74] M. A. L Thathachar and P. S. Sastry. A Class of Rapidly Converging Algorithms for Learning Automata. IEEE Transactions on Systems, Man and Cybernetics, 15:168-175, 1985.

[75] M. A. L Thathachar and PS Sastry. A New Approach to the Design of Reinforcement Schemes for Learning Automata. IEEE transactions on systems, man, and cybernetics, 15(1):168-175, 1985.

[76] M. L. Tsetlin. On the Behavior of Finite Automata in Random Media. Avtomatika i Telemekhanika, 22:1345-1354, 1961.

[77] C. Unsal, P. Kachroo, and J.S. Bay. Multiple stochastic learning automata for vehicle path control in an automated highway system. Systems, Man and 
Cybernetics, Part A: Systems and Humans, IEEE Transactions on, 29(1):120$128,1999$.

[78] V. I. Varshavskii and I. P. Vorontsova. On the Behavior of Stochastic Automata with Variable Structure. Automation and Remote Control, 24(3), 1963.

[79] P. Verkhogliad and B. J. Oommen. Potential AI Strategies to Solve the Commons Game: A Position Paper. In Advances in Artificial Intelligence: 23rd Canadian Conference on Artificial Intelligence, pages 352-356. Springer, 2010.

[80] P. Verkhogliad and B. J. Oommen. Using Artificial Intelligence Techniques for Strategy Generation in the Commons Game. In The 6th International Conference on Hybrid Artificial Intelligence Systems. (To appear), 2011.

[81] Y. Xing, C. N. Mathur, M. A. Haleem, R. Chandramouli, and K. P. Subbalakshmi. Dynamic Spectrum Access with QoS and Interference Temperature Constraints. IEEE Transactions on Mobile Computing, pages 423-433, 2007.

[82] X. Yao and P. J. Darwen. An Experimental Study of N-Person Iterated Prisoner's Dilemma Games. Informatica, 18:435-450, 1994. 